IJMMS 32:12 (2002) 739-761

PII. S0161171202007019

http://ijmms.hindawi.com

(c) Hindawi Publishing Corp.

\title{
PARAMETRICALLY EXCITED NONLINEAR SYSTEMS: A COMPARISON OF TWO METHODS
}

\author{
A. F. EL-BASSIOUNY \\ Received 9 March 2001
}

\begin{abstract}
Subharmonic resonance of two-degree-of-freedom systems with cubic nonlinearities to multifrequency parametric excitations in the presence of three-to-one internal resonance is investigated. Two approximate methods (the multiple scales and the generalized synchronization) are used to construct a first-order nonlinear ordinary differential equations governing the modulation of the amplitudes and phases. Steady state solutions and their stability are computed for selected values of the system parameters. The results obtained by the two methods are in excellent agreement. Numerical solutions are carried out and graphical representations of the results are presented and discussed.
\end{abstract}

2000 Mathematics Subject Classification: 65P30, 37M20, 37G15.

1. Introduction. In many practical situations, the load on a structure can be modeled by a finite sum of temporarily harmonic terms. For example, such a situation occurs when a single structure supports several different rotating machines. In other situations, the load acting on a structure, although periodic, is not harmonic but can be expressed as the sum of a finite number of harmonic terms.

Some curious phenomena can develop as a result of the interactions of the different harmonics. These can be recognized and understood by studying the response of structure elements, such as beams and plates, to multiple harmonic loads.

Although problems involving single-frequency excitations have received considerable attention $[1,11,12,13,15,17]$, limited problems involving multifrequency excitations have been studied in [2, 19] for the case of one-degree-of-freedom systems and in $[3,4,5,7,8,14,16,18]$ for the case of multidegree-of-freedom systems.

In [2], the method of multiple scales was used to account for all these nonlinear corrections to the natural frequency. Yamamoto et al. [19] examined the response of single-degree-of-freedom systems with cubic nonlinearities to combination resonances. Plaut et al. [18] studied the influence of an internal resonance on nonlinear structural vibrations under two frequency excitations. Asfar et al. [3] studied the response of single-degree-of-freedom self-excited oscillators to multifrequency excitations. Nayfeh [16] considered the nonlinear response of a bowed structure to combination resonances. Ashworth and Barr [5] investigated the resonances of structures with quadratic inertial nonlinearity under direct and parametric harmonic excitation. Mojaddidy et al. [14] examined the response of a beam to a combination resonant excitation in the presence and absence of internal resonance. Asfar et al. [4] examined the response of two-degree-of-freedom self-excited oscillators to multifrequency 
excitations. In [8], the method of multiple scales was used to studied harmonic, subharmonic, superharmonic, simultaneous sub/superharmonic and combination resonances of self-excited two coupled second-order system to multifrequency excitations. In [7], the response of self-excited three-degree-of-freedom systems to multifrequency excitations was analyzed.

This paper is concerned with response of two-degree-of-freedom systems with cubic nonlinearities to multifrequency parametric excitations governed by the equations

$$
\begin{aligned}
\ddot{X}_{1}+\omega_{1}^{2} X_{1}+\epsilon[ & 2 \mu_{1} \dot{X}_{1}+\alpha_{1} X_{1}^{3}+3 \alpha_{2} X_{1}^{2} X_{2}+\alpha_{3} X_{1} X_{2}^{2}+\alpha_{4} X_{2}^{3} \\
& \left.+2 \sum_{m=1}^{M}\left\{X_{1} f_{1 m}+X_{2} f_{2 m}\right\} \cos \left(\Omega_{m} t+\tau_{1 m}\right)\right]=0, \\
\ddot{X}_{2}+\omega_{2}^{2} X_{2}+\epsilon[ & 2 \mu_{2} \dot{X}_{2}+\alpha_{2} X_{1}^{3}+3 \alpha_{3} X_{1}^{2} X_{2}+\alpha_{4} X_{1} X_{2}^{2}+\alpha_{5} X_{2}^{3} \\
& \left.+2 \sum_{n=1}^{N}\left\{X_{1} g_{1 n}+X_{2} g_{2 n}\right\} \cos \left(\Omega_{n} t+\gamma_{1 n}\right)\right]=0,
\end{aligned}
$$

where $\omega_{n}, \mu_{n}, \alpha_{n}, f_{m n}, g_{m n}, \Omega_{m}, \Omega_{n}, \tau_{1 m}$, and $\gamma_{1 n}$ are constants, $\epsilon$ is a small dimensionless parameter, and dots indicate differentiation with respect to the time $t$. These equations when quadratic terms are included, model the responses of ships and bowed structural elements. Two approximate methods (the multiple scales and the generalized synchronization) are used to comparison. The modulation equations (reduced equations) of the amplitudes and phases are obtained. Steady state solutions and their stability are determined. Numerical calculations are carried out. Graphical representation of the results are presented. Discussion of the figures is given. The results obtained by two methods are in excellent agreement.

2. The method of multiple scales (see [15]). To determine a first-order uniform solution of (1.1), we use the method of multiple scales and let

$$
X(t ; \epsilon)=X_{n 0}\left(T_{0}, T_{1}\right)+\epsilon X_{n 1}\left(T_{0}, T_{1}\right)+\cdots,
$$

where $T_{0}=t$ is a fast scale, which is associated with changes occurring at the frequencies $\omega_{n}, \Omega_{m}$, and $\Omega_{n}$ and $T_{1}=\epsilon t$ is a slow scale, which is associated with modulations in the amplitudes and phases resulting from the nonlinearities and parametric resonances. In terms of $T_{0}$ and $T_{1}$ the time derivative become

$$
\frac{d}{d t}=D_{0}+\epsilon D_{1}+\cdots, \quad \frac{d^{2}}{d t^{2}}=D_{0}^{2}+2 \epsilon D_{0} D_{1}+\cdots,
$$

where $D_{n}=\partial / \partial T_{n}$. Substituting (2.1) and (2.2) into (1.1) and equating coefficients of power $\epsilon$, we obtain

$$
D_{0}^{2} X_{10}+\omega_{1}^{2} X_{10}=0, \quad D_{0} X_{20}^{2}+\omega_{1} X_{20}=0,
$$




$$
\begin{aligned}
D_{0}^{2} X_{11}+\omega_{1}^{2} X_{11}= & -2 D_{0} D_{1} X_{10}-2 \mu_{1}\left(D_{0} X_{10}\right)-\alpha_{1} X_{10}^{3}-3 \alpha_{2} X_{10}^{2} X_{20}-3 \alpha_{3} X_{10} X_{20}^{2} \\
& -\alpha_{4} X_{20}^{3}+2 \sum_{m=1}^{M}\left\{X_{10} f_{1 m}+X_{20} f_{2 m}\right\} \cos \left(\Omega_{m} t+\tau_{1 m}\right) \\
D_{0}^{2} X_{21}+\omega_{2}^{2} X_{21}= & -2 D_{0} D_{1} X_{20}-2 \mu_{2}\left(D_{0} X_{20}\right)-\alpha_{2} X_{10}^{3}-3 \alpha_{3} X_{10}^{2} X_{20}-3 \alpha_{4} X_{10} X_{20}^{2} \\
& -\alpha_{5} X_{20}^{3}+2 \sum_{n=1}^{N}\left\{X_{10} g_{1 n}+X_{20} g_{2 n}\right\} \cos \left(\Omega_{n} t+v_{1 n}\right) .
\end{aligned}
$$

The solution of (2.3) can be expressed as

$$
X_{10}=A_{1} \exp \left(i \omega_{1} T_{0}\right)+c c, \quad X_{20}=A_{2} \exp \left(i \omega_{1} T_{0}\right)+c c
$$

where $c c$ denotes the complex conjugate of the preceding terms. Inserting (2.6) into (2.5) yields

$$
\begin{aligned}
& D_{0}^{2} X_{11}+\omega_{1}^{2} X_{11}=\left[-2 i \omega_{1}\left(A^{\prime}+\mu_{1} A_{1}\right)+3 \alpha_{1} A_{10}^{2} \bar{A}_{10}+6 \alpha_{3} A_{1} A_{2} A_{2} \bar{A}_{2}\right] \exp \left(i \omega_{1} T_{0}\right) \\
& -\left[6 \alpha_{2} A_{1} \bar{A}_{1} A_{2}+3 \alpha_{4} A_{2}^{2} \bar{A}_{2}\right] \exp \cdot\left(2 i \omega_{2} T_{0}\right) \\
& -\alpha_{1} A_{1}^{3} \exp \left(3 i \omega_{1} T_{0}\right)-\alpha_{4} A_{2}^{3} \exp \left(3 i \omega_{2} T_{0}\right) \\
& -3 \alpha_{2}\left[A_{1}^{2} A_{2} \exp i\left(2 \omega_{1}+\omega_{2}\right) T_{0}+\bar{A}_{1}^{2} A_{2} \exp i\left(\omega_{2}-2 \omega_{1}\right) T_{0}\right] \\
& -3 \alpha_{3}\left[A_{1} A_{2}^{2} \exp i\left(\omega_{1}+2 \omega_{2}\right) T_{0}+A_{1} \bar{A}_{2}^{2} \exp i\left(\omega_{1}-2 \omega_{2}\right) T_{0}\right. \\
& \left.+\bar{A}_{1} A_{2}^{2} \exp i\left(2 \omega_{2}-\omega_{1}\right) T_{0}\right] \\
& -A_{1} \sum_{m=1}^{M} f_{1 m} \exp \left(\left(\Omega_{m}+\omega_{1}\right) T_{0}+\tau_{1 m}\right) \\
& -\bar{A}_{1} \sum_{m=1}^{M} f_{1 m} \exp \left(\left(\Omega_{m}-\omega_{1}\right) T_{0}+\tau_{1 m}\right) \\
& -A_{2} \sum_{m=1}^{M} f_{2 m} \exp \left(\left(\Omega_{m}+\omega_{2}\right) T_{0}+\tau_{1 m}\right) \\
& -\bar{A}_{2} \sum_{m=1}^{M} f_{2 m} \exp \left(\left(\Omega_{m}-\omega_{2}\right) T_{0}+\tau_{1 m}\right), \\
& D_{0}^{2} X_{21}+\omega_{2}^{2} X_{21}=-\left[2 i \omega_{2}\left(A_{2}^{\prime}+\mu_{2} A_{2}\right)+6 \alpha_{3} A_{1} \bar{A}_{1} A_{2}+3 \alpha_{5} \bar{A}_{2}^{2} A_{2}\right] \exp \left(i \omega_{2} T_{0}\right) \\
& \text { - }\left[3 \alpha_{2} A_{1}^{2} \bar{A}_{1}+6 \alpha_{4} A_{2} \bar{A}_{2}\right] \exp \left(i \omega_{1} T_{0}\right) \\
& -\alpha_{2} A_{1}^{3} \exp \left(3 i \omega_{1} T_{0}\right)-\alpha_{5} A_{2}^{3} \exp \left(3 i \omega_{2} T_{0}\right) \\
& -3 \alpha_{4}\left[A_{1} A_{2}^{2} \exp i\left(\omega_{1}+2 \omega_{2}\right) T_{0}+A_{1} \bar{A}_{2}^{2} \exp i\left(\omega_{1}-2 \omega_{2}\right) T_{0}\right. \\
& \left.+\bar{A}_{1}^{2} A_{2}^{2} \exp i\left(\omega_{2}+2 \omega_{1}\right) T_{0}\right]
\end{aligned}
$$




$$
\begin{aligned}
& -A_{1} \sum_{n=1}^{N} g_{1 n} \exp \left(\left(\Omega_{n}+\omega_{1}\right) T_{0}+\gamma_{1 n}\right) \\
& +\bar{A}_{1} \sum_{n=1}^{N} g_{1 n} \exp \left(\left(\Omega_{n}-\omega_{1}\right) T_{0}+\gamma_{1 n}\right) \\
& -A_{2} \sum_{n=1}^{N} g_{2 n} \exp \left(\left(\Omega_{n}+\omega_{2}\right) T_{0}+v_{1 n}\right) \\
& -\bar{A}_{2} \sum_{n=1}^{N} f_{2 m} \exp \left(\left(\Omega_{n}-\omega_{2}\right) T_{0}+v_{1 n}\right),
\end{aligned}
$$

where the overbar indicates the complex conjugate, and the prime indicates differentiation with respect to $T_{1}$. Any particular solution of (2.7) contain secular or small divisor terms depending on the resonant conditions (a) $\omega_{2} \cong 2 \omega_{1}$, internal resonance; (b) $\Omega_{r} \cong 2 \omega_{1}$, principal parametric resonance of the first mode. To treat this case, we introduce a detuning parameters $\sigma_{1}$ and $\sigma_{2}$ to convert the small divisor terms into secular terms, defined according to

$$
\omega_{2}=2 \omega_{1}+\epsilon \sigma_{1}, \quad \Omega_{r}=2 \omega_{1}+\epsilon \sigma_{2} .
$$

Substituting (2.8) into (2.7) and eliminating the secular terms from $X_{11}$ and $X_{21}$, we obtain

$$
\begin{aligned}
& 2 i \omega_{1}\left(A_{1}^{\prime}+\mu_{1} A_{1}\right)+3 \alpha_{1} A_{1}^{2} \bar{A}_{1}+6 \alpha_{3} A_{1} A_{2} \bar{A}_{2} \\
& \quad+3 \alpha_{2} \bar{A}_{1}^{2} A_{2} \exp \left(i \sigma_{1} T_{1}\right)+\bar{A}_{1} f_{1 r} \exp i\left(\sigma_{2} T_{1}+\tau_{1 r}\right)=0, \\
& 2 i \omega_{2}\left(A_{2}^{\prime}+\mu_{2} A_{2}\right)+6 \alpha_{3} A_{1} \bar{A}_{1} A_{2}+3 \alpha_{5} A_{2}^{2} \bar{A}_{2}+\alpha_{2} A_{1}^{3} \exp \left(-i \sigma_{1} T_{1}\right)=0 .
\end{aligned}
$$

Consequently, the particular solutions of (2.7) are

$$
\begin{aligned}
U_{11}= & -\left[\frac{6 \alpha_{2} A_{1} \bar{A}_{1} A_{2}+3 \alpha_{4} A_{2}^{2} \bar{A}_{2}}{\omega_{1}^{2}-\omega_{2}^{2}}\right] \exp \left(i \omega_{2} T_{0}\right) \\
& +\left[\frac{\alpha_{1} A_{1}^{3}}{8 \omega_{1}^{2}}\right] \exp \left(3 i \omega_{1} T_{0}\right)-\left[\frac{\alpha_{4} A_{2}^{3}}{\omega_{1}^{2}-9 \omega_{2}^{2}}\right] \exp \left(3 i \omega_{2} T_{0}\right) \\
& -\left[\frac{3 \alpha_{2} A_{1}^{2} A_{2}}{\left\{\omega_{1}^{2}-\left(2 \omega_{1}+\omega_{2}\right)^{2}\right\}}\right] \exp i\left(2 \omega_{1}+\omega_{2}\right) T_{0} \\
& -\left[\frac{3 \alpha_{2} \bar{A}_{1}^{2} A_{2}}{\left\{\omega_{1}^{2}-\left(\omega_{2}-2 \omega_{1}\right)^{2}\right\}}\right] \exp i\left(\omega_{2}-2 \omega_{1}\right) T_{0} \\
& -\left[\frac{3 \alpha_{3} A_{1} A_{2}^{2}}{\left\{\omega_{1}^{2}-\left(\omega_{1}+2 \omega_{2}\right)^{2}\right\}}\right] \exp i\left(\omega_{1}+2 \omega_{2}\right) T_{0}
\end{aligned}
$$




$$
\begin{aligned}
& -\left[\frac{3 \alpha_{3} A_{1} \bar{A}_{2}^{2}}{\left\{\omega_{1}^{2}-\left(\omega_{1}-2 \omega_{2}\right)^{2}\right\}}\right] \exp i\left(\omega_{1}-2 \omega_{2}\right) T_{0} \\
& -\left[\frac{3 \alpha_{3} \bar{A}_{1} A_{2}^{2}}{\left\{\omega_{1}^{2}-\left(2 \omega_{2}-\omega_{1}\right)^{2}\right\}}\right] \exp i\left(2 \omega_{2}-\omega_{1}\right) T_{0} \\
& -\left[\frac{f_{1 r} A_{1}}{\left\{\omega_{1}^{2}-\left(\Omega_{r}+\omega_{1}+\tau_{1 r}\right)^{2}\right\}}\right] \exp i\left(\left(\Omega_{r}+\omega_{1}\right) T_{0}+\tau_{1 r}\right) \\
& -\left[\frac{f_{2 r} A_{2}}{\left\{\omega_{1}^{2}-\left(\Omega_{r}+\omega_{2}+\tau_{2 r}\right)^{2}\right\}}\right] \exp i\left(\left(\Omega_{r}+\omega_{2}\right) T_{0}+\tau_{1 r}\right) \\
& -\left[\frac{f_{2 r} \bar{A}_{2}}{\left\{\omega_{1}^{2}-\left(\Omega_{r}-\omega_{2}+\tau_{2 r}\right)^{2}\right\}}\right] \exp i\left(\left(\Omega_{r}-\omega_{2}\right) T_{0}+\tau_{1 r}\right) \text {, } \\
& U_{21}=-\left[\frac{3 \alpha_{2} A_{1}^{2} \bar{A}_{1}+6 \alpha_{4} A_{1} A_{2} \bar{A}_{2}}{\omega_{2}^{2}-\omega_{1}^{2}}\right] \exp \left(i \omega_{1} T_{0}\right) \\
& -\left[\frac{\alpha_{2} A_{1}^{3}}{\omega_{2}^{2}-9 \omega_{1}^{2}}\right] \exp \left(3 i \omega_{1} T_{0}\right)+\left[\frac{\alpha_{5} A_{2}^{3}}{8 \omega_{2}^{2}}\right] \exp \left(3 i \omega_{2} T_{0}\right) \\
& -\left[\frac{3 \alpha_{3} A_{1}^{2} A_{2}}{\left\{\omega_{2}^{2}-\left(2 \omega_{1}-\omega_{2}\right)^{2}\right\}}\right] \exp i\left(2 \omega_{1}+\omega_{2}\right) T_{0} \\
& -\left[\frac{3 \alpha_{3} \bar{A}_{1}^{2} A_{2}}{\left\{\omega_{2}^{2}-\left(\omega_{2}-2 \omega_{1}\right)^{2}\right\}}\right] \exp i\left(\omega_{2}-2 \omega_{1}\right) T_{0} \\
& -\left[\frac{3 \alpha_{4} A_{1} A_{2}^{2}}{\left\{\omega_{2}^{2}-\left(\omega_{1}+2 \omega_{2}\right)^{2}\right\}}\right] \exp i\left(\omega_{1}+2 \omega_{2}\right) T_{0} \\
& -\left[\frac{3 \alpha_{4} A_{1} \bar{A}_{2}^{2}}{\left\{\omega_{2}^{2}-\left(\omega_{1}-2 \omega_{2}\right)^{2}\right\}}\right] \exp i\left(\omega_{1}-2 \omega_{2}\right) T_{0} \\
& -\left[\frac{3 \alpha_{4} \bar{A}_{1} A_{2}^{2}}{\left\{\omega_{2}^{2}-\left(2 \omega_{2}-\omega_{1}\right)^{2}\right\}}\right] \exp i\left(2 \omega_{2}-\omega_{1}\right) T_{0} \\
& -\left[\frac{g_{1 s} A_{1}}{\left\{\omega_{2}^{2}-\left(\Omega_{s}+\omega_{1}+\gamma_{1 s}\right)^{2}\right\}}\right] \exp i\left(\left(\Omega_{s}+\omega_{1}\right) T_{0}+\gamma_{1 s}\right) \\
& -\left[\frac{g_{1 s} \bar{A}_{1}}{\left\{\omega_{2}^{2}-\left(\Omega_{s}-\omega_{1}+\gamma_{1 s}\right)^{2}\right\}}\right] \exp i\left(\left(\Omega_{s}+\omega_{1}\right) T_{0}+\gamma_{1 s}\right) \\
& -\left[\frac{g_{2 r} A_{2}}{\left\{\omega_{2}^{2}-\left(\Omega_{s}+\omega_{2}+\gamma_{2 s}\right)^{2}\right\}}\right] \exp i\left(\left(\Omega_{s}+\omega_{2}\right) T_{0}+\gamma_{1 s}\right) \text {. }
\end{aligned}
$$

Expressing $A_{n}$ in the polar notation

$$
A_{n}=\frac{1}{2} a_{n} \exp \left(i \beta_{n}\right)
$$


and separating the real and imaginary parts of (2.9), we obtain

$$
\begin{aligned}
a_{1}^{\prime} & =-\mu_{1} a_{1}-\frac{3 \alpha_{2}}{8 \omega_{1}} a_{1}^{2} a_{2} \sin \theta_{1}-\frac{1}{2 \omega_{1}} f_{1 r} a_{1} \sin \theta_{2}, \\
a_{1} \beta_{1}^{\prime} & =\frac{3 \alpha_{1}}{8 \omega_{1}} a_{1}^{3}+\frac{3 \alpha_{3}}{4 \omega_{1}} a_{1} a_{2}^{2}+\frac{3 \alpha_{2}}{8 \omega_{1}} a_{1}^{2} a_{2} \cos \theta_{1}+\frac{1}{2 \omega_{1}} f_{1 r} a_{1} \cos \theta_{2}, \\
a_{2}^{\prime} & =-\mu_{2} a_{2}+\frac{\alpha_{2}}{8 \omega_{2}} a_{1}^{3} \sin \theta_{1}, \\
a_{2} \beta_{2}^{\prime} & =\frac{3 \alpha_{3}}{4 \omega_{2}} a_{1}^{2} a_{2}+\frac{3 \alpha_{5}}{8 \omega_{2}} a_{2}^{3}+\frac{\alpha_{2}}{8 \omega_{2}} a_{1}^{3} \cos \theta_{1},
\end{aligned}
$$

where,

$$
\theta_{1}=\sigma_{1} T_{1}+\beta_{2}-3 \beta_{1}, \quad \theta_{2}=\sigma_{2} T_{1}-2 \beta_{1}+\tau_{1 r} .
$$

Inserting (2.6) and (2.10) into (2.1) yield the approximate solutions

$$
\begin{aligned}
& U_{1}=a_{1} \cos \left(\omega_{1} t+\beta_{1}\right) \\
& +\epsilon\left[-\left[\frac{6 \alpha_{2} a_{1}^{2} a_{2}+3 \alpha_{4} a_{2}^{3}}{4 \omega_{1}^{2}-4 \omega_{2}^{2}}\right] \cos \psi_{2}\right. \\
& +\left[\frac{\alpha_{1} a_{1}^{3}}{32 \omega_{1}^{2}}\right] \cos 3 \psi_{1}-\left[\frac{\alpha_{4} a_{2}^{3}}{4\left(\omega_{1}^{2}-9 \omega_{2}^{2}\right)}\right] \cos 3 \psi_{2} \\
& -\left[\frac{3 \alpha_{2} a_{1}^{2} a_{2}}{\left\{4 \omega_{1}^{2}-4\left(2 \omega_{1}+\omega_{2}\right)^{2}\right\}}\right] \cos \left(2 \psi_{1}+\psi_{2}\right) \\
& -\left[\frac{3 \alpha_{2} a_{1}^{2} a_{2}}{\left\{4 \omega_{1}^{2}-4\left(2 \omega_{1}+\omega_{2}\right)^{2}\right\}}\right] \cos \left(\psi_{2}-2 \psi_{1}\right) \\
& -\left[\frac{3 \alpha_{3} a_{1} a_{2}^{2}}{\left\{4 \omega_{1}^{2}-4\left(\omega_{1}+2 \omega_{2}\right)^{2}\right\}}\right] \cos \left(\psi_{1}+2 \psi_{2}\right) \\
& -\left[\frac{3 \alpha_{3} a_{1} a_{2}^{2}}{\left\{4 \omega_{1}^{2}-4\left(\omega_{1}-2 \omega_{2}\right)^{2}\right\}}\right] \cos \left(\psi_{1}-2 \psi_{2}\right) \\
& -\left[\frac{3 \alpha_{3} a_{1} a_{2}^{2}}{\left\{4 \omega_{1}^{2}-4\left(2 \omega_{2}-\omega_{1}\right)^{2}\right\}}\right] \cos \left(2 \psi_{2}-\psi_{1}\right) \\
& -\left[\frac{a_{1} f_{1 m}}{\left\{\omega_{1}^{2}-\left(\Omega_{r}+\omega_{1} \psi_{1 r}\right)^{2}\right\}}\right] \cos \left(\psi_{3}+\psi_{1}\right) \\
& -\left[\frac{a_{2} f_{2 r}}{\left\{\omega_{1}^{2}-\left(\Omega_{r}+\omega_{2}+\psi_{2 r}\right)^{2}\right\}}\right] \cos \left(\psi_{3}+\psi_{2}\right) \\
& \left.-\left[\frac{a_{2} f_{2 r}}{\left\{\omega_{1}^{2}-\left(\Omega_{r}-\omega_{2}+\psi_{2 r}\right)^{2}\right\}}\right] \cos \left(\psi_{4}-\psi_{2}\right)\right]+o\left(\epsilon^{2}\right) \text {, }
\end{aligned}
$$




$$
\begin{aligned}
& U_{2}=a_{2} \cos \left(\omega_{2} t+\beta_{2}\right) \\
& +\epsilon\left[\left[\frac{3 \alpha_{2} a_{1}^{3}+6 \alpha_{4} a_{1} a_{2}^{2}}{4 \omega_{2}^{2}-4 \omega_{1}^{2}}\right] \cos \psi_{1}\right. \\
& -\left[\frac{\alpha_{2} a_{1}^{3}}{\omega_{2}^{2}-9 \omega_{1}^{2}}\right] \cos 3 \psi_{2}+\left[\frac{\alpha_{5} a_{2}^{3}}{32 \omega_{2}^{2}}\right] \cos 3 \psi_{2} \\
& -\left[\frac{3 \alpha_{3} a_{1}^{2} a_{2}}{\left\{4 \omega_{2}^{2}-4\left(\omega_{2}-2 \omega_{1}\right)^{2}\right\}}\right] \cos \left(\psi_{1}+2 \psi_{2}\right) \\
& -\left[\frac{3 \alpha_{3} a_{1}^{2} a_{2}}{\left\{4 \omega_{2}^{2}-4\left(\omega_{2}-2 \omega_{1}\right)^{2}\right\}}\right] \cos \left(\psi_{2}-2 \psi_{1}\right) \\
& -\left[\frac{3 \alpha_{4} a_{1} a_{2}^{2}}{\left\{4 \omega_{2}^{2}-4\left(\omega_{1}+2 \omega_{2}\right)^{2}\right\}}\right] \cos \left(\psi_{1}+2 \psi_{2}\right) \\
& -\left[\frac{3 \alpha_{4} a_{1} a_{2}^{2}}{\left\{4 \omega_{2}^{2}-4\left(\omega_{1}-2 \omega_{2}\right)^{2}\right\}}\right] \cos \left(\psi_{1}-2 \psi_{2}\right) \\
& -\left[\frac{3 \alpha_{4} a_{1} a_{2}^{2}}{\left\{4 \omega_{2}^{2}-4\left(2 \omega_{2}-\omega_{1}\right)^{2}\right\}}\right] \cos \left(2 \psi_{2}-\psi_{1}\right) \\
& -\left[\frac{g_{1 s} a_{1}}{\left\{\omega_{2}^{2}-\left(\Omega_{s}+\omega_{1}+\gamma_{1 s}\right)^{2}\right\}}\right] \cos \left(\psi_{4}+\psi_{1}\right) \\
& -\left[\frac{g_{1 s} a_{1}}{\left\{\omega_{2}^{2}-\left(\Omega_{s}-\omega_{1}+\gamma_{1 s}\right)^{2}\right\}}\right] \cos \left(\psi_{4}-\psi_{1}\right) \\
& \left.-\left[\frac{g_{2 r} a_{2}}{\left\{\omega_{2}^{2}-\left(\Omega_{s}+\omega_{2}+\gamma_{2 s}\right)^{2}\right\}}\right] \cos \left(\psi_{4}+\psi_{2}\right)\right]+o\left(\epsilon^{2}\right) \text {, }
\end{aligned}
$$

where,

$$
\psi_{1}=\omega_{1} t \beta_{1}, \quad \psi_{2}=\omega_{2} t+\beta_{2}, \quad \psi_{3}=\Omega_{r} t+\tau_{1 \gamma}, \quad \psi_{4}=\Omega_{s} t+\gamma_{1 n} .
$$

The steady state of (2.12) are given by

$$
\begin{aligned}
& \mu_{1} a_{1}+\frac{3 \alpha_{2}}{8 \omega_{1}} a_{1}^{2} a_{2} \sin \theta_{1}+\frac{1}{2 \omega_{1}} f_{1 r} a_{1} \sin \theta_{2}=0, \\
& \frac{1}{2} \delta_{2} a_{1}-\frac{3 \alpha_{1}}{8 \omega_{1}} a_{1}^{3}-\frac{3 \alpha_{3}}{4 \omega_{1}} a_{1} a_{2}^{2}-\frac{3 \alpha_{2}}{8 \omega_{1}} a_{1}^{2} a_{2} \cos \theta_{1}-\frac{1}{2 \omega_{1}} f_{1 r} a_{1} \cos \theta_{2}=0, \\
& -\mu_{2} a_{2}+\frac{3 \alpha_{2}}{8 \omega_{1}} a_{1}^{2} a_{2} \sin \theta_{1}=0, \\
& \left(\frac{3}{2} \delta_{2}-\sigma_{1}\right) a_{2}+\frac{3 \alpha_{3}}{4 \omega_{2}} a_{1} a_{2}^{2}+\frac{3 \alpha_{5}}{8 \omega_{2}} a_{2}^{3}+\frac{3 \alpha_{2}}{8 \omega_{1}} a_{1}^{2} a_{2} \cos \theta_{1}=0 .
\end{aligned}
$$


There are two possibilities: first, $a_{1}=a_{2}=0$; this is the linear case; second, $a_{1}$ and $a_{2} \neq 0$ and (2.16) yield the frequency response equations

$$
\begin{aligned}
\mu_{1}^{2}+ & \frac{1}{4} \delta_{2}^{2}+\left[\frac{9 \alpha_{2}^{2}}{64 \omega_{1}^{2}}\right] a_{1}^{2} a_{2}^{2}+\left[\frac{9 \alpha_{1}^{2}}{64 \omega_{1}^{2}}\right] a_{1}^{4}-\frac{1}{4 \omega_{1}^{2}} f_{1 r}^{2} \\
+ & {\left[\frac{3 \alpha_{2} \mu_{1}}{4 \omega_{1}}\right] a_{1} a_{2} \sin \theta_{1}+\left[\frac{3 \alpha_{1} \sigma_{2}}{8 \omega_{1}}\right] a_{1}^{2} } \\
- & {\left[\frac{3 \alpha_{3} \sigma_{2}}{4 \omega_{1}}\right] a_{2}^{2}-\left[\frac{3 \alpha_{2} \sigma_{2}}{8 \omega_{1}}\right] a_{1} a_{2} \cos \theta_{1}+\left[\frac{9 \alpha_{1} \alpha_{2}}{16 \omega_{1}^{2}}\right] a_{1}^{2} a_{2}^{2} } \\
+ & {\left[\frac{9 \alpha_{1} \alpha_{2}}{32 \omega_{1}^{2}}\right] a_{1}^{3} a_{2} \cos \theta_{1}+\left[\frac{9 \alpha_{2} \alpha_{3}}{16 \omega_{1}^{2}}\right] a_{1} a_{2}^{3} \cos \theta_{1}=0, } \\
{\left[\mu_{2}^{2}+\right.} & \left.\left(\frac{3}{2} \delta_{2}-\delta_{1}\right)^{2}\right] a_{2}^{2}-\left[\frac{\alpha_{2}^{2}}{64 \omega_{2}^{2}}\right] a_{1}^{6}+\left[\frac{9 \alpha_{3}^{2}}{16 \omega_{2}^{2}}\right] a_{1}^{4} a_{2}^{2}+\frac{9 \alpha_{5}^{2}}{64 \omega_{2}^{2}} a_{2}^{6} \\
& -\left[\frac{3 \alpha_{3}}{2 \omega_{2}}\left(\frac{3}{2} \delta_{2}-\delta_{1}\right)\right] a_{1}^{2} a_{2}^{2}-\left[\frac{3 \alpha_{5}}{4 \omega_{2}}\left(\frac{3}{2} \delta_{2}-\delta_{1}\right)\right] a_{2}^{4} \\
+ & {\left[\frac{3 \alpha_{3} \alpha_{5}}{32 \omega_{2}}\right] a_{1}^{2} a_{2}^{4}=0 . }
\end{aligned}
$$

3. Stability analysis. Here we study the problem of stability in two cases: trivial and nontrivial solutions.

3.1. Trivial solution and its stability. To study the stability of the trivial solution, we find it convenient to express $A_{n}$ in the Cartesian form

$$
A_{n}=\frac{1}{2}\left[p_{n}\left(T_{1}\right)-q_{n}\left(T_{1}\right)\right] \exp \left(i v_{n} T_{1}\right),
$$

where

$$
v_{1}=\frac{1}{2} \sigma_{2}, \quad v_{2}=\frac{3}{2} \sigma_{2}-\sigma_{1} .
$$

Inserting (3.1) into (2.9), and separating the real and imaginary parts, we obtain

$$
\begin{gathered}
p_{1}^{\prime}+\mu_{1} p_{1}+q_{1} \nu_{1}-\frac{3 \alpha_{1}}{8 \omega_{1}}\left(p_{1}^{2} q_{1}+q_{1}^{3}\right) \\
-\frac{3 \alpha_{3}}{4 \omega_{1}}\left(p_{2}^{2} q_{1}+q_{1} q_{2}^{2}\right)+\frac{1}{\omega_{1}} f_{1 r} q_{1} \cos \tau_{1 r}=0 \\
q_{1}^{\prime}+\mu_{1} q_{1}-p_{1} v_{1}-\frac{3 \alpha_{1}}{8 \omega_{1}}\left(p_{1}^{2} q_{1}+q_{1}^{3}\right) \\
+\frac{3 \alpha_{3}}{4 \omega_{1}}\left(p_{1}^{2} q_{1}+q_{1}^{3}\right)+\frac{1}{\omega_{1}} f_{1 r} p_{1} \cos \tau_{1 r}=0, \\
p_{2}^{\prime}+\mu_{2} p_{2}+q_{2} v_{2}-\frac{3 \alpha_{3}}{4 \omega_{2}}\left(p_{1}^{2} q_{2}+q_{1}^{2} q_{2}\right)-\frac{3 \alpha_{5}}{8 \omega_{2}}\left(p_{2}^{2} q_{2}+q_{2}^{3}\right)=0, \\
q_{2}^{\prime}+\mu_{2} q_{2}-p_{2} v_{2}-\frac{3 \alpha_{3}}{4 \omega_{2}}\left(p_{1}^{2} p_{2}+q_{1}^{2} p_{2}\right)+\frac{3 \alpha_{5}}{8 \omega_{2}}\left(p_{2}^{3}+p_{2} q_{2}^{2}\right)=0 .
\end{gathered}
$$


The trivial solution corresponds to $p_{n}^{\prime}=q_{n}^{\prime}=0$ for $n=1,2$. The stability of this solution can be determined by the eigenvalues of the Jacobian matrix of (3.3) evaluated at $p_{n}=q_{n}=0$. They are given by

$$
\lambda^{4}+r_{1} \lambda^{3}+r_{2} \lambda^{2}+r_{3} \lambda+r_{4}=0
$$

where

$$
\begin{aligned}
& r_{1}=2\left(\mu_{1}+\mu_{2}\right), \\
& r_{2}=\mu_{1}^{2}+2 \mu_{1} \mu_{2}+\mu_{2}^{2}+v_{1}^{2}+v_{2}^{2}-\frac{1}{\omega_{1}^{2}} f_{1 r}^{2} \cos ^{2} \psi_{1 r}, \\
& r_{3}=2 \mu_{1} \mu_{2}\left(\mu_{1}+\mu_{2}\right)+2\left(\mu_{1} v_{2}^{2}+\mu_{2} v_{1}^{2}\right)-2 \mu_{2} \frac{1}{\omega_{1}^{2}} f_{1 r}^{2} \cos ^{2} \psi_{1 r}, \\
& r_{4}=\mu_{1}^{2} \mu_{2}^{2}-\mu_{1}^{2} v_{2}^{2}+\mu_{2}^{2} v_{1}^{2}-\mu_{2}^{2} \frac{1}{\omega_{1}^{2}} f_{1 r}^{2} \cos ^{2} \psi_{1 r}+v_{2}^{2}\left[\frac{1}{\omega_{1}^{2}} f_{1 r}^{2} \cos ^{2} \psi_{1 r}-v_{1}^{2}\right] .
\end{aligned}
$$

According to the Routh-Hurwitz criterion, the solution is stable if and only if the following conditions are satisfied:

$$
r_{1}>0, \quad\left[r_{1} r_{2}-r_{3}\right]>0, \quad\left[r_{3}\left(r_{1} r_{2}-r_{3}\right)-r_{1}^{2} r_{4}\right]>0, \quad r_{4}>0
$$

The stable (unstable) solutions are represented by solid (dashed) lines on $\sigma_{2}$-axis.

3.2. Stability of nontrivial solution. To determine the stability of the nontrivial solution, let

$$
a_{i}=a_{i 0}+a_{i 1}, \quad \theta_{i}=\theta_{i 0}+\theta_{i 1} \quad(i=1,2),
$$

where $a_{i 0}, \theta_{i 0}$ are the steady state values, and $a_{i 1}$ and $\theta_{i 1}$ are infinitesimal timedependent perturbation. Inserting (3.7) into (2.12), using (2.16), and linearizing the resulting equations, we get

$$
\begin{aligned}
a_{11}^{\prime}= & {\left[\mu_{1}+\frac{3 \alpha_{2}}{4 \omega_{1}} a_{10} a_{20} \sin \theta_{10}+\frac{1}{2 \omega_{1}} f_{1 r} \sin \theta_{20}\right] a_{11} } \\
& -\frac{3 \alpha_{2}}{8 \omega_{1}} a_{10}^{2} \sin \theta_{10} a_{21}-\left[\frac{3 \alpha_{2}}{8 \omega_{1}} a_{10}^{2} a_{20} \cos \theta_{10}\right] \theta_{11}-\left[\frac{1}{2 \omega_{1}} f_{1 r} a_{10} \cos \theta_{20}\right] \theta_{21}, \\
a_{21}^{\prime}= & {\left[\frac{3 \alpha_{2}}{8 \omega_{2}} a_{10}^{2} \sin \theta_{10}\right] a_{11}-\mu_{2} a_{21}+\frac{3 \alpha_{2}}{8 \omega_{2}} a_{10}^{3} \cos \theta_{10} \theta_{11}, } \\
\theta_{11}^{\prime}= & {\left[\frac{\sigma_{1}}{a_{10}}+\frac{3 \alpha_{2}}{8 \omega_{1}} a_{10}^{2} \cos \theta_{10}+\left\{\frac{3 \alpha_{3}}{2 \omega_{2}}\right\} a_{10}-\left\{\frac{9 \alpha_{1}}{4 \omega_{1}}\right\} a_{10}\right.} \\
& \left.-\left\{\frac{9 \alpha_{3} a_{20}^{2}}{4 \omega_{2} a_{10}}\right\}-\frac{1}{\omega_{1} a_{10}} f_{1 r} \cos \theta_{20}-\frac{9 \alpha_{2}}{8 \omega_{2}} a_{10}^{2} \cos \theta_{10}\right] a_{11}
\end{aligned}
$$




$$
\begin{aligned}
+ & {\left[\left\{\frac{3 \alpha_{3} a_{10}}{4 \omega_{2} a_{20}}\right\}+\left\{\frac{9 \alpha_{5}}{8 \omega_{2}}\right\} a_{20}^{2}-\left\{\frac{9 \alpha_{3}}{2 \omega_{1}}\right\} a_{20}+\frac{9 \alpha_{2}}{8 \omega_{1}} a_{10}^{2} \cos \theta_{10}\right] a_{21} } \\
& +\left[\frac{3 \alpha_{2}}{4 \omega_{1}} a_{10}^{2} a_{20} \sin \theta_{10}+\frac{3 \alpha_{2}}{8 \omega_{1}} a_{10} a_{20} \sin \theta_{10}\right] \theta_{11}-\left[\frac{1}{2 \omega_{1}} f_{1 r} \sin \theta_{20}\right] \theta_{21} \\
\theta_{21}^{\prime}= & {\left[\left\{\frac{9 \alpha_{1}}{4 \omega_{1}}\right\} a_{10}+\left\{\frac{3 \alpha_{3} a_{20}^{2}}{2 \omega_{1} a_{10}}\right\}+\frac{1}{\omega_{1} a_{10}} f_{1 r} \cos \theta_{20}+\frac{3 \alpha_{2}}{2 \omega_{1}} a_{10} a_{20} \cos \theta_{10}\right] a_{11} } \\
& +\left[\frac{\sigma_{1}}{a_{10}}-\left\{\frac{3 \alpha_{2}}{\omega_{1}}\right\} a_{20}-\frac{3 \alpha_{2}}{4 \omega_{1}} a_{10}^{2} \cos \theta_{10}\right] a_{21}+\left[\frac{3 \alpha_{2}}{4 \omega_{1}} a_{10}^{2} a_{20} \sin \theta_{10}\right] \theta_{11} \\
& +\left[\frac{1}{2 \omega_{1}} f_{1 r} \sin \theta_{20}\right] \theta_{21} .
\end{aligned}
$$

Put $a_{i 1}=c_{i} \exp \left(\lambda T_{1}\right)$ and $\theta_{i 1}=c_{i+2} \exp \left(\lambda T_{1}\right)$ into (3.6) and (3.8). The solution is stable if and only if the real part of each of the eigenvalues of the coefficient of the matrix is less than zero. The stable solutions are represented by solid lines and the unstable solutions by broken lines on the frequency response curves.

4. The generalized synchronization method (see [6, 9]). When $\epsilon=0$, the general solution of (1.1) are

$$
X_{n}=a_{n} \cos \left(\omega_{n} t+\phi_{n}\right), \quad \dot{X}_{n}=-\omega_{n} a_{n} \cos \left(\omega_{n} t+\phi_{n}\right) \quad(n=1,2) .
$$

For $\epsilon \neq 0$ small enough, let $a_{n}$ and $\phi_{n}$ be unknown functions of time $t$ in (1.1). By differentiating the first equation of (4.1) and equating with the second, and differentiating the second equation of (4.1), we obtain

$$
\dot{a}_{n} \cos \left(\omega_{n} t+\phi_{n}\right)-a_{n} \omega_{n} \sin \left(\omega_{n} t+\phi_{n}\right)=0
$$

By using (4.1) and (4.2), (1.1) are reduced to a system of first-order differential equations in the variables $a_{n}(t)$ and $\phi_{n}(t)$ as follows:

$$
\begin{aligned}
\dot{a}_{1}(t)=\epsilon[ & -\mu_{1} a_{1}\left(1-\cos \psi_{1}\right)+\frac{\alpha_{1}}{4 \omega_{1}} a_{1}^{3}\left[\sin 2 \psi_{1}+\frac{1}{2} \sin 4 \psi_{1}\right] \\
& +\frac{\alpha_{2}}{4 \omega_{1}} a_{1}^{2} a_{2}\left[\sin \left(3 \psi_{1}+\psi_{2}\right)+\sin \left(3 \psi_{1}-\psi_{2}\right)+\sin \left(\psi_{1}+\psi_{2}\right)+\sin \left(\psi_{1}-\psi_{2}\right)\right] \\
& +\frac{3 \alpha_{3}}{2 \omega_{1}} a_{1} a_{2}^{2}\left[\sin \left(2 \psi_{2}+2 \psi_{1}\right)+\sin \left(2 \psi_{2}-2 \psi_{1}\right)\right] \\
& +\frac{\alpha_{4}}{8 \omega_{1}} a_{2}^{3}\left[\sin \left(3 \psi_{2}+\psi_{1}\right)+\frac{1}{2} \sin \left(3 \psi_{2}-\psi_{1}\right)\right. \\
& \left.\quad+3 \sin \left(\psi_{2}+\psi_{1}\right)+3 \sin \left(\psi_{2}-\psi_{1}\right)\right] \\
& +\frac{1}{2 \omega_{1}} a_{1} f_{1 r}\left[\sin \left(\psi_{3}+2 \psi_{1}\right)-\sin \left(\psi_{3}-2 \psi_{1}\right)\right]
\end{aligned}
$$




$$
\begin{aligned}
& +\frac{1}{2 \omega_{1}} a_{2} f_{2 r}\left[\sin \left(\psi_{4}+\psi_{2}+\psi_{1}\right)+\sin \left(\psi_{4}+\psi_{2}-\psi_{1}\right)\right. \\
& \left.\left.+\sin \left(\psi_{4}-\psi_{2}+\psi_{1}\right)-\sin \left(\psi_{4}-\psi_{2}-\psi_{1}\right)\right]\right], \\
& \dot{\phi}_{1}(t)=\epsilon\left[\mu_{1} \sin 2 \psi_{1}+\frac{\alpha_{1}}{8 \omega_{1}} a_{1}^{2}\left[\cos 4 \psi_{1}+4 \cos 2 \psi_{1}+3\right]\right. \\
& +\frac{3 \alpha_{2}}{8 \omega_{1}} a_{1} a_{2}\left[\cos \left(3 \psi_{1}+\psi_{2}\right)+\cos \left(3 \psi_{1}-\psi_{2}\right)\right. \\
& \left.+3 \cos \left(\psi_{1}+\psi_{2}\right)+3 \cos \left(\psi_{1}-\psi_{2}\right)\right] \\
& +\frac{3 \alpha_{3}}{4 \omega_{1}} a_{2}^{2}\left[1+\cos 2 \psi_{2}+\cos 2 \psi_{1}+\frac{1}{2}\left\{\cos \left(2 \psi_{1}+2 \psi_{2}\right)+\cos \left(2 \psi_{1}-2 \psi_{2}\right)\right\}\right] \\
& +\left\{\frac{\alpha_{4} a_{2}^{3}}{8 \omega_{1} a_{1}}\right\}\left[\cos \left(3 \psi_{2}+\psi_{1}\right)+\cos \left(3 \psi_{2}-\psi_{1}\right)\right. \\
& \left.+3 \cos \left(\psi_{2}+\psi_{1}\right)+3 \cos \left(\psi_{2}-\psi_{1}\right)\right] \\
& +\frac{1}{2 \omega_{1}} f_{1 r}\left[\cos \left(\psi_{3}+2 \psi_{1}\right)+2 \cos 2 \psi_{3}+\cos \left(\psi_{3}-2 \psi_{1}\right)\right] \\
& +\left\{\frac{a_{2}}{2 \omega_{1} a_{1}}\right\} f_{2 r}\left[\cos \left(\psi_{4}+\psi_{2}+\psi_{1}\right)+\cos \left(\psi_{4}+\psi_{2}-\psi_{1}\right)\right. \\
& \left.\left.+\cos \left(\psi_{4}-\psi_{2}+\psi_{1}\right)+\cos \left(\psi_{4}-\psi_{2}-\psi_{1}\right)\right]\right], \\
& \dot{a}_{2}(t)=\epsilon\left[-\mu_{2} a_{2}\left(1-\cos \psi_{2}\right)+\frac{\alpha_{2}}{8 \omega_{2}} a_{1}^{3}\left[\sin \left(3 \psi_{1}-\psi_{2}\right)+\frac{1}{2} \sin \left(3 \psi_{1}+\psi_{2}\right)\right.\right. \\
& \left.+3 \sin \left(\psi_{1}+\psi_{2}\right)-3 \sin \left(\psi_{1}-\psi_{2}\right)\right] \\
& +\frac{3 \alpha_{3}}{8 \omega_{2}} a_{1}^{2} a_{2}\left[\sin \left(2 \psi_{1}+2 \psi_{2}\right)+\sin \left(2 \psi_{1}-2 \psi_{2}\right)\right] \\
& +\frac{3 \alpha_{4}}{8 \omega_{2}} a_{1} a_{2}^{2}\left[\sin \left(3 \psi_{2}+\psi_{1}\right)+\sin \left(3 \psi_{2}-\psi_{1}\right)+\sin \left(\psi_{2}+\psi_{1}\right)+\sin \left(\psi_{2}-\psi_{1}\right)\right] \\
& +\frac{\alpha_{5}}{4 \omega_{2}} a_{2}^{3}\left[\sin 2 \psi_{2}+\frac{1}{2} \sin 4 \psi_{2}\right] \\
& +\frac{1}{2 \omega_{2}} a_{1} g_{1 s}\left[\sin \left(\psi_{5}+\psi_{1}+\psi_{2}\right)-\sin \left(\psi_{5}+\psi_{1}-\psi_{2}\right)\right. \\
& \left.+\sin \left(\psi_{5}-\psi_{1}+\psi_{2}\right)-\sin \left(\psi_{5}-\psi_{1}-\psi_{2}\right)\right] \\
& \left.+\frac{1}{2 \omega_{2}} a_{2} g_{2 s}\left[\sin \left(\psi_{6}+2 \psi_{2}\right)+\sin \left(\psi_{6}-2 \psi_{2}\right)\right]\right], \\
& \dot{\phi}_{2}(t)=\epsilon\left[\mu_{2} \sin 2 \psi_{2}+\left\{\frac{\alpha_{2} a_{1}^{3}}{8 \omega_{2} a_{2}}\right\}\left[\cos \left(3 \psi_{1}+\psi_{2}\right)+\cos \left(3 \psi_{1}-\psi_{2}\right)\right.\right. \\
& \left.+3 \cos \left(\psi_{1}+\psi_{2}\right)+3 \cos \left(\psi_{1}-\psi_{2}\right)\right]
\end{aligned}
$$




$$
\begin{aligned}
& +\frac{3 \alpha_{3}}{4 \omega_{2}} a_{1}^{2}\left[1+\cos 2 \psi_{2}+\cos 2 \psi_{1}+\frac{1}{2}\left\{\cos \left(2 \psi_{1}+2 \psi_{2}\right)+\cos \left(2 \psi_{1}-2 \psi_{2}\right)\right\}\right] \\
& +\frac{3 \alpha_{4}}{8 \omega_{2}} a_{1} a_{2}\left[\cos \left(3 \psi_{2}+\psi_{1}\right)+\cos \left(3 \psi_{2}-\psi_{1}\right)\right. \\
& \left.\quad+3 \cos \left(\psi_{2}+\psi_{1}\right)+\cos \left(\psi_{2}-\psi_{1}\right)\right] \\
& +\frac{\alpha_{5}}{8 \omega_{2}} a_{2}^{3}\left[\cos 4 \psi_{2}+4 \cos 2 \psi_{2}+3\right] \\
& +\left\{\frac{a_{1}}{2 \omega_{2} a_{2}}\right\} g_{1 s}\left[\cos \left(\psi_{5}+\psi_{1}+\psi_{2}\right)+\cos \left(\psi_{5}+\psi_{1}-\psi_{2}\right)\right. \\
& \left.\quad+\cos \left(\psi_{5}-\psi_{1}+\psi_{2}\right)+\cos \left(\psi_{5}-\psi_{1}-\psi_{2}\right)\right] \\
& \left.+\frac{1}{2 \omega_{2}} g_{2 s}\left[\cos \left(\psi_{6}+2 \psi_{2}\right)+\cos \left(\psi_{6}-2 \psi_{2}\right)\right]\right]
\end{aligned}
$$

For principal parametric synchronization of the first mode $\left(\Omega_{r} \cong 2 \omega_{1}\right)$ in the presence of internal synchronization $\left(\omega_{2} \cong 3 \omega_{1}\right)$, then from (4.3) we retain only the constant terms and the terms of small frequency. Then, we obtain

$$
\bar{F}(y, t)=f(y, t)=\left[\begin{array}{ll}
f_{1 a} & (y, t) \\
f_{1 \phi} & (y, t) \\
f_{2 a} & (y, t) \\
f_{2 \phi} & (y, t)
\end{array}\right], \quad y=\left[\begin{array}{l}
A_{1} \\
\phi_{1} \\
A_{2} \\
\phi_{2}
\end{array}\right],
$$

where,

$$
\begin{aligned}
\bar{f}_{1 a}(y, t)= & -\mu_{1} A_{1}+\frac{3 \alpha_{2}}{8 \omega_{1}} A_{1}^{2} A_{2} \sin \left[\left(3 \omega_{1}-\omega_{2}\right) t+3 \Phi_{1}-\Phi_{2}\right] \\
& -\frac{1}{2 \omega_{1}} A_{1} f_{1 r} \sin \left[\left(\Omega_{r}-2 \omega_{1}\right) t-\left(2 \Phi_{1}-\tau_{1 r}\right)\right] \\
\bar{f}_{1 \phi}(y, t)= & {\left[\frac{3 \alpha_{1}}{8 \omega_{1}}\right] A_{1}^{2}+\left[\frac{3 \alpha_{3}}{4 \omega_{1}}\right] A_{2}^{2}+\frac{3 \alpha_{2}}{8 \omega_{1}} A_{1} A_{2} \cos \left[\left(3 \omega_{1}-\omega_{2}\right) t+3 \Phi_{1}-\Phi_{2}\right] } \\
& +\frac{1}{2 \omega_{1}} f_{1 r} \cos \left[\left(\Omega_{r}-2 \omega_{1}\right) t-\left(2 \Phi_{1}-\tau_{1 r}\right)\right] \\
\bar{f}_{2 a}(y, t)= & -\mu_{2} A_{2}+\frac{\alpha_{2}}{8 \omega_{2}} A_{1}^{3} \sin \left[\left(3 \omega_{1}-\omega_{2}\right) t+3 \Phi_{1}-\Phi_{2}\right] \\
\bar{f}_{2 \phi}(y, t)= & {\left[\frac{3 \alpha_{3}}{4 \omega_{2}}\right] A_{1}^{2}+\left[\frac{3 \alpha_{5}}{8 \omega_{2}}\right] A_{2}^{2} } \\
& +\frac{\alpha_{2} A_{1}^{3}}{8 \omega_{2} A_{2}} \cos \left[\left(3 \omega_{1}-\omega_{2}\right) t+3 \Phi_{1}-\Phi_{2}\right]
\end{aligned}
$$


and the term of higher frequency is

$$
\tilde{f}(y, t)=\left[\begin{array}{c}
\tilde{f}_{1 a}(y, t) \\
\tilde{f}_{1 \phi}(y, t) \\
\tilde{f}_{2 a}(y, t) \\
\tilde{f}_{2 \phi}(y, t)
\end{array}\right],
$$

where,

$$
\begin{aligned}
& \tilde{f}_{1 a}(y, t)=\mu_{1} a_{1} \cos 2 \psi_{1}+\frac{\alpha_{1}}{4 \omega_{1}} a_{1}^{3}\left[\sin 2 \psi_{1}+\frac{1}{2} \sin 4 \psi_{1}\right] \\
& +\frac{3 \alpha_{2}}{8 \omega_{1}} a_{1}^{2} a_{2}\left[\sin \left(3 \psi_{1}+\psi_{2}\right)+\sin \left(\psi_{1}+\psi_{2}\right)+\sin \left(\psi_{1}-\psi_{2}\right)\right] \\
& +\frac{3 \alpha_{3}}{8 \omega_{1}} a_{1} a_{2}^{2}\left[\sin \left(2 \psi_{2}+2 \psi_{1}\right)+\sin \left(2 \psi_{2}-2 \psi_{1}\right)\right] \\
& +\frac{\alpha_{4}}{8 \omega_{1}} a_{2}^{3}\left[\sin \left(3 \psi_{2}+\psi_{1}\right)+\frac{1}{2} \sin \left(3 \psi_{2}-\psi_{1}\right)\right. \\
& \left.+\sin \left(\psi_{2}+\psi_{1}\right)+\sin \left(\psi_{2}-\psi_{1}\right)\right] \\
& +\frac{1}{2 \omega_{1}} a_{1} f_{1 r} \sin \left(\psi_{3}+2 \psi_{1}\right) \\
& +\frac{1}{2 \omega_{1}} a_{2} f_{2 r}\left[\sin \left(\psi_{4}+\psi_{2}+\psi_{1}\right)-\sin \left(\psi_{4}+\psi_{2}-\psi_{1}\right)\right. \\
& \left.+\sin \left(\psi_{4}-\psi_{2}+\psi_{1}\right)-\sin \left(\psi_{4}-\psi_{2}-\psi_{1}\right)\right], \\
& \tilde{f}_{1 \phi}(y, t)=-\mu_{1} \sin 2 \psi_{1}+\frac{\alpha_{1}}{8 \omega_{1}} a_{1}^{2}\left[\cos 4 \psi_{1}+4 \cos 2 \psi_{1}\right] \\
& +\frac{3 \alpha_{2}}{8 \omega_{1}} a_{1} a_{2}\left[\cos \left(3 \psi_{1}+\psi_{2}\right)+3 \cos \left(\psi_{1}+\psi_{2}\right)+3 \cos \left(\psi_{1}-\psi_{2}\right)\right] \\
& +\frac{3 \alpha_{3}}{4 \omega_{1}} a_{2}^{2}\left[\cos 2 \psi_{2}+\cos 2 \psi_{1}+\frac{1}{2}\left\{\cos \left(2 \psi_{1}+2 \psi_{2}\right)+\cos \left(2 \psi_{1}-2 \psi_{2}\right)\right\}\right] \\
& +\left\{\frac{\alpha_{4} a_{2}^{3}}{8 \omega_{1} a_{1}}\right\}\left[\cos \left(3 \psi_{2}+\psi_{1}\right)+\cos \left(3 \psi_{2}-\psi_{1}\right)\right. \\
& \left.+3 \cos \left(\psi_{2}+\psi_{1}\right)+3 \cos \left(\psi_{2}-\psi_{1}\right)\right] \\
& +\frac{1}{2 \omega_{1}} f_{1 r}\left[\cos \left(\psi_{3}+2 \psi_{1}\right)+2 \cos \psi_{3}\right] \\
& +\left\{\frac{a_{2}}{2 \omega_{1} a_{1}}\right\} f_{2 r}\left[\cos \left(\psi_{4}+\psi_{2}+\psi_{1}\right)+\cos \left(\psi_{4}+\psi_{2}-\psi_{1}\right)\right. \\
& \left.+\cos \left(\psi_{4}-\psi_{2}+\psi_{1}\right)+\cos \left(\psi_{4}-\psi_{2}-\psi_{1}\right)\right] \\
& \tilde{f}_{2 a}(y, t)=-\mu_{2} a_{2}\left(1-\cos \psi_{2}\right) \\
& +\frac{\alpha_{2}}{8 \omega_{2}} a_{1}^{3}\left[\frac{1}{2} \sin \left(3 \psi_{1}+\psi_{2}\right)+3 \sin \left(\psi_{1}+\psi_{2}\right)-3 \sin \left(\psi_{1}-\psi_{2}\right)\right]
\end{aligned}
$$




$$
\begin{aligned}
+ & \frac{3 \alpha_{3}}{8 \omega_{2}} a_{1}^{2} a_{2}\left[\sin \left(2 \psi_{1}+2 \psi_{2}\right)+\sin \left(2 \psi_{1}-2 \psi_{2}\right)\right] \\
+ & \frac{3 \alpha_{4}}{8 \omega_{2}} a_{1} a_{2}^{2}\left[\sin \left(3 \psi_{2}+\psi_{1}\right)+\sin \left(3 \psi_{2}-\psi_{1}\right)\right. \\
& \left.\quad+\sin \left(\psi_{2}+\psi_{1}\right)+\sin \left(\psi_{2}-\psi_{1}\right)\right] \\
+ & \frac{\alpha_{5}}{4 \omega_{2}} a_{2}^{3}\left[\sin 2 \psi_{2}+\frac{1}{2} \sin 4 \psi_{2}\right] \\
+ & \frac{1}{2 \omega_{2}} a_{1} g_{1 s}\left[\sin \left(\psi_{5}+\psi_{1}+\psi_{2}\right)-\sin \left(\psi_{5}+\psi_{1}-\psi_{2}\right)+\sin \left(\psi_{5}-\psi_{1}+\psi_{2}\right)\right. \\
\tilde{f}_{2 \phi}(y, t)= & \mu_{2} \sin _{2} \psi_{2}+\left\{\frac{\alpha_{2} a_{1}^{3}}{8 \omega_{2} a_{2}}\right\}\left[\sin \left(\psi_{5}-\psi_{1}-\psi_{2}\right)\right]+\frac{1}{2 \omega_{2}} a_{2} g_{2 s} \sin \left(\psi_{6}+2 \psi_{2}\right) \\
+ & \frac{3 \alpha_{3}}{4 \omega_{2}} a_{1}^{2}\left[\cos 2 \psi_{2}+\cos 2 \psi_{1}+\frac{1}{2}\left\{\cos \left(2 \psi_{1}+2 \psi_{2}\right)+\cos \left(2 \psi_{1}-2 \psi_{2}\right)\right\}\right] \\
+ & \frac{3 \alpha_{4}}{8 \omega_{2}} a_{1} a_{2}\left[\cos \left(3 \psi_{2}+\psi_{1}\right)+\cos \left(3 \psi_{2}-\psi_{1}\right)\right.
\end{aligned}
$$

Then the reduced system to the first approximation takes the form

$$
\left[\begin{array}{l}
A_{1} \\
\phi_{1} \\
A_{2} \\
\phi_{2}
\end{array}\right]=\epsilon \bar{F}(y, t) .
$$

Then, we get

$$
\begin{aligned}
\dot{A}_{1}=\epsilon[ & -\mu_{1} A_{1}-\frac{3 \alpha_{2}}{8 \omega_{1}} A_{1}^{2} A_{2} \sin \left[\left(3 \omega_{1}-\omega_{2}\right) t+3 \Phi_{1}-\Phi_{2}\right] \\
& \left.-\frac{1}{2 \omega_{1}} A_{1} f_{1 r} \sin \left[\left(\Omega_{r}-2 \omega_{1}\right) t-\left(2 \Phi_{1}-\psi_{1 r}\right)\right]\right] \\
\dot{\Phi}_{1}=\epsilon[ & {\left[\frac{3 \alpha_{1}}{8 \omega_{1}}\right] A_{1}^{2}+\left[\frac{3 \alpha_{3}}{4 \omega_{1}}\right] A_{2}^{2}+\left\{\frac{3 \alpha_{2}}{8 \omega_{2}}\right\} A_{1} A_{2} \cos \left[\left(3 \omega_{1}-\omega_{2}\right) t+3 \Phi_{1}-\Phi_{2}\right] } \\
& \left.+\frac{1}{2 \omega_{1}} f_{1 r} \cos \left[\left(\Omega_{r}-2 \omega_{1}\right) t-\left(2 \Phi_{1}-\psi_{1 r}\right)\right]\right]
\end{aligned}
$$




$$
\begin{aligned}
& \dot{A}_{2}=\epsilon\left[-\mu_{2} A_{2}-\frac{\alpha_{2}}{8 \omega_{2}} A_{1}^{3} \sin \left[\left(3 \omega_{1}-\omega_{2}\right) t+3 \Phi_{1}-\Phi_{2}\right]\right], \\
& \dot{\Phi}_{2}=\epsilon\left[\left[\frac{3 \alpha_{3}}{4 \omega_{2}}\right] A_{1}^{2}+\left[\frac{3 \alpha_{5}}{8 \omega_{2}}\right] A_{2}^{2}+\frac{\alpha_{2} A_{1}^{2}}{8 \omega_{2} A_{2}} \cos \left[\left(3 \omega_{1}-\omega_{2}\right) t+3 \Phi_{1}-\Phi_{2}\right]\right] .
\end{aligned}
$$

Since $t$ appears explicitly in (4.9), they are called a nonatonomous system. It is convenient to eliminate the explicit dependence on $t$, thereby transforming these equations into an autonomous system. This can be accomplished by introducing the new dependent variables $\gamma_{1}$ and $\gamma_{2}$ defined by

$$
\gamma_{1}=\left(\omega_{2}-3 \omega_{1}\right) t+\Phi_{2}-3 \Phi_{1}, \quad \gamma_{2}=\left(\Omega_{s}-2 \omega_{1}\right) t-2 \Phi_{1}+\tau_{1 r} .
$$

Inserting (4.10) into (4.9), we obtain the autonomous system that describe the modulation of the amplitudes and the phases

$$
\begin{aligned}
\dot{A}_{1}= & \epsilon\left[-\mu_{1} A_{1}-\frac{3 \alpha_{2}}{8 \omega_{1}} A_{1}^{2} A_{2} \sin \gamma_{1}-\frac{1}{2 \omega_{1}} A_{1} f_{1 r} \sin \gamma_{2}\right], \\
\frac{1}{2}\left[\epsilon \sigma_{2}-\dot{\gamma}_{2}\right]= & \epsilon\left[\left\{\frac{3 \alpha_{1}}{8 \omega_{1}}\right\} A_{1}^{2}+\left\{\frac{3 \alpha_{3}}{4 \omega_{1}}\right\} A_{2}^{2}\right] \\
+ & \epsilon\left[\left\{\frac{3 \alpha_{2} A_{1}^{3}}{8 \omega_{2} A_{2}}\right\} \cos \gamma_{1}+\frac{1}{2 \omega_{1}} f_{1 r} \cos \gamma_{1}\right], \\
\dot{A}_{2}= & \epsilon\left[-\mu_{2} A_{2}+\frac{\alpha_{2}}{8 \omega_{2}} A_{1}^{3} \sin \gamma_{2}-\frac{1}{2 \omega_{2}} A_{2} g_{2 s} \sin \gamma_{2}\right], \\
{\left[\epsilon\left(\frac{3}{2} \sigma_{2}-\sigma_{1}\right)+\dot{\gamma}_{1}-\frac{3}{2} \dot{\gamma}_{2}\right]=} & \epsilon\left[\frac{3 \alpha_{3}}{4 \omega_{2}}\right] A_{1}^{2}+\epsilon\left[\left[\frac{3 \alpha_{5}}{8 \omega_{2}}\right] A_{2}^{2}+\frac{\alpha_{2} A_{1}^{3}}{8 \omega_{2} A_{2}} \cos \gamma_{2}\right] .
\end{aligned}
$$

For the system of (4.11) to have stationary solutions, the following conditions must be satisfied

$$
\dot{A}_{n}=\dot{\gamma}_{n}=0 .
$$

Hence, the steady state solutions are given by

$$
\begin{gathered}
\mu_{1} A_{1}+\frac{3 \alpha_{2}}{8 \omega_{1}} A_{1}^{2} A_{2} \sin \gamma_{1}+\frac{1}{2 \omega_{1}} A_{1} f_{1 r} \sin \gamma_{1}=0 \\
\frac{1}{2} \sigma_{2}-\left\{\frac{3 \alpha_{1}}{8 \omega_{1}}\right\} A_{1}^{2}-\left\{\frac{3 \alpha_{3}}{4 \omega_{1}}\right\} A_{2}^{2}-\left\{\frac{3 \alpha_{2} A_{1}^{3}}{8 \omega_{2} A_{2}}\right\} \cos \gamma_{1}-\frac{1}{2 \omega_{1}} f_{1 r} \cos \gamma_{1}=0 \\
-\mu_{2} A_{2}+\frac{\alpha_{2}}{8 \omega_{2}} A_{1}^{3} \sin \gamma_{2}=0 \\
\left(\frac{3}{2} \sigma_{2}-\sigma_{1}\right)-\left[\frac{3 \alpha_{3}}{4 \omega_{2}}\right] A_{1}^{2}+\frac{3 \alpha_{5}}{8 \omega_{2}} A_{2}^{2}+\frac{\alpha_{2} A_{1}^{3}}{8 \omega_{2} A_{2}} \cos \gamma_{2}=0
\end{gathered}
$$

These equations are in full agreement with (2.16) obtained using the method of multiple scales. Also, from these equations we can obtain the frequency response equations, which are similar to (2.17). 
In order to establish the approximate amplitudes and approximate phases, we find the function $G(y, t)$ as follows:

$$
\tilde{G}(y, t)=\left[\begin{array}{c}
\tilde{G}_{1 a}(y, t) \\
\tilde{G}_{1 \phi}(y, t) \\
\tilde{G}_{1 a}(y, t) \\
\tilde{G}_{2 \phi}(y, t)
\end{array}\right],
$$

where,

$$
\begin{array}{ll}
\tilde{G}_{1 a}(y, t)=\int \tilde{f}_{1 a}(y, t) d t, & \tilde{G}_{1 \phi}(y, t)=\int \tilde{f}_{1 \phi}(y, t) d t, \\
\tilde{G}_{2 a}(y, t)=\int \tilde{f}_{2 a}(y, t) d t, & \tilde{G}_{2 \phi}(y, t)=\int \tilde{f}_{2 \phi}(y, t) d t .
\end{array}
$$

Then, the approximate solutions are

$$
\begin{aligned}
& U_{1}(t)=a_{1}(t) \cos \left[\omega_{1} t+\phi_{1}(t)\right]=\left[A_{1}+\epsilon \tilde{G}_{1 a}(y, t)\right] \cos \left[\omega_{1} t+\Phi_{1}(t)+\epsilon \tilde{G}_{1 \phi}(y, t)\right] \\
& =A_{1} \cos \psi_{1}-\epsilon\left[\left\{\frac{\mu_{1} A_{1}}{4 \omega_{1}}\right\}\left(\sin \psi_{1}+\sin 3 \psi_{1}\right)-\frac{3}{32 \omega_{1}^{2}}\left[2 \alpha_{1} A_{1}^{3}+\alpha_{3} A_{1} A_{2}^{2}\right] \cos \psi_{1}\right. \\
& +\left[\frac{6 \alpha_{2} A_{1}^{2} A_{2}+3 \alpha_{4} A_{2}^{3}}{8 \omega_{1}^{2}-8 \omega_{2}^{2}}\right] \cos \psi_{2}-\frac{1}{32 \omega_{1}^{2}}\left[\alpha_{1} A_{1}^{3}+3 \alpha_{3} A_{1} A_{2}^{2}\right] \cos 3 \psi_{1} \\
& -\left\{\frac{2 \alpha_{4}\left(3 \omega_{2}-\omega_{1}\right)}{16 \omega_{1}\left(9 \omega_{2}^{2}-\omega_{1}^{2}\right)}\right\} A_{2}^{3} \cos 3 \psi_{2} \\
& -\frac{3}{16 \omega_{1}}\left[\frac{6 \alpha_{2} \omega_{2} A_{1}^{2} A_{2}}{9 \omega_{1}^{2}-\omega_{2}^{2}}+\frac{\alpha_{2} A_{1}^{2} A_{2}}{3 \omega_{1}+\omega_{2}}+\left(\frac{\alpha_{3} A_{2}^{3}}{\omega_{2}-\omega_{1}}\right)\right] \cos \left(\psi_{2}-\psi_{1}\right) \\
& -\left\{\frac{3 \alpha_{3}\left(5 \omega_{2}+\omega_{1}\right) A_{1} A_{2}^{2}}{32 \omega_{1} \omega_{2}\left(3 \omega_{1}+\omega_{2}\right)}\right\} \cos \left(\psi_{1}+2 \psi_{2}\right) \\
& -\left\{\frac{3 \alpha_{3}\left(5 \omega_{2}-\omega_{1}\right) A_{1} A_{2}^{2}}{32 \omega_{1} \omega_{2}\left(\omega_{2}-\omega_{1}\right)}\right\} \cos \left(\psi_{1}-2 \psi_{2}\right) \\
& -\left\{\frac{3 \alpha_{2} A_{1}^{2} A_{2}}{16 \omega_{1}\left(3 \omega_{1}+\omega_{2}\right)}\right\} \cos \left(4 \psi_{1}+\psi_{2}\right) \\
& -\left\{\frac{3 \alpha_{2} A_{1} A_{2}}{16 \omega_{1}\left(3 \omega_{1}-\omega_{2}\right)}\right\} \cos \left(4 \psi_{1}-\psi_{2}\right) \\
& -\left\{\frac{3 \alpha_{3} A_{1} A_{2}^{2}}{8 \omega_{1}\left(\omega_{1}+\omega_{2}\right)}\right\} \cos \left(2 \psi_{2}+3 \psi_{1}\right) \\
& -\left\{\frac{15 \alpha_{3} A_{1} A_{2}^{2}}{64 \omega_{1}\left(\omega_{2}-\omega_{1}\right)}\right\} \cos \left(2 \psi_{2}-3 \psi_{1}\right) \\
& -\left\{\frac{\alpha_{4} A_{2}^{3}}{8 \omega_{1}\left(3 \omega_{2}-\omega_{1}\right)}\right\} \cos \left(3 \psi_{2}-2 \psi_{1}\right)
\end{aligned}
$$




$$
\begin{aligned}
& +\left\{\frac{1}{\Omega_{r}\left(\Omega_{r}+2 \omega_{1}\right)}\right\} A_{1} f_{1 r} \cos \left(\psi_{3}+2 \psi_{1}\right) \\
& -\left\{\frac{1}{4 \omega_{1} \Omega_{r}}\right\} A_{1} f_{1 r} \cos \left(\psi_{3}-\psi_{1}\right) \\
& +\left\{\frac{1}{2 \omega_{1}\left(\Omega_{r}+\omega_{2}+\omega_{1}\right)}\right\} A_{2} f_{2 r} \cos \left(\psi_{3}+\psi_{2}\right) \\
& \left.+\left\{\frac{1}{\left(\Omega_{r}-\omega_{2}-\omega_{1}\right)\left(\Omega_{r}-\omega_{2}+\omega_{1}\right)}\right\} A_{2} f_{2 r} \cos \left(\psi_{3}-\psi_{2}\right)\right],
\end{aligned}
$$

$U_{2}(t)=a_{2}(t) \cos \left[\omega_{2} t+\phi_{2}(t)\right]=\left[A_{2}+\epsilon \tilde{G}_{2 a}(y, t)\right] \cos \left[\omega_{2} t+\Phi_{2}(t)+\epsilon \tilde{G}_{2 \phi}(y, t)\right]$

$$
\begin{aligned}
& =A_{2} \cos \psi_{2}-\epsilon\left[\left\{\frac{\mu_{2} A_{2}}{2 \omega_{2}}\right\} \sin 3 \psi_{2}\right. \\
& -\frac{3 \alpha_{3} A_{1}^{2} A_{2}}{16 \omega_{2}}\left[\frac{1}{\omega_{1}+\omega_{2}}-6\left\{\frac{1}{4\left(\omega_{1}+\omega_{2}\right)}-\frac{1}{4\left(\omega_{1}-\omega_{2}\right)}\right\}\right] \cos \psi_{1} \\
& -\frac{1}{16 \omega_{1}^{2}}\left\{\alpha_{5} A_{2}^{3}+3 \alpha_{3} A_{1}^{2} A_{2}\right\} \cos \psi_{2} \\
& -\frac{\alpha_{2} A_{1}^{2}}{8 \omega_{2}^{2}}\left\{\frac{2}{3 \omega_{1}-\omega_{2}}+\frac{1}{3 \omega_{1}+\omega_{2}}\right\} \cos 3 \psi_{1} \\
& -\frac{1}{64 \omega_{2}^{2}}\left\{-\alpha_{5} A_{2}^{3}+12 \alpha_{3} A_{1}^{2} A_{2}\right\} \cos 3 \psi_{2}-\frac{\alpha_{5} A_{2}^{3}}{64 \omega_{2}^{2}} \cos 5 \psi_{2} \\
& +\left[-\left\{\frac{3 \alpha_{3} A_{1}^{2} A_{2}}{32 \omega_{2}\left(\omega_{1}+\omega_{2}\right)}\right\}+\left\{\frac{3 \alpha_{4} A_{1} A_{2}^{2}}{4 \omega_{2}\left(\omega_{1}+\omega_{2}\right)}\right\}\right. \\
& \left.+\left\{\frac{3 \alpha_{4} A_{1} A_{2}^{2}}{8 \omega_{2}\left(\omega_{1}+3 \omega_{2}\right)}\right\}\right] \cos \left(\psi_{1}+2 \psi_{2}\right) \\
& +\left\{\frac{3 \alpha_{4} A_{1} A_{2}^{2}}{8 \omega_{2}\left(3 \omega_{2}+\omega_{1}\right)}\right\} \cos \left(\psi_{1}+4 \psi_{2}\right) \\
& -\frac{3 \alpha_{3} A_{1}^{2} A_{2}}{16 \omega_{2}}\left\{\frac{1}{\omega_{1}-\omega_{2}}+\frac{1}{2 \omega_{1}}\right\} \cos \left(2 \psi_{1}-\psi_{2}\right) \\
& -\frac{3 \alpha_{4} A_{1} A_{2}^{2}}{16 \omega_{2}}\left\{\frac{1}{\omega_{2}-\omega_{1}}-\frac{1}{3 \omega_{2}+\omega_{1}}\right\} \cos \left(2 \psi_{2}-\psi_{1}\right) \\
& -\left\{\frac{\alpha_{2} A_{1}^{3}}{16 \omega_{2}\left(3 \omega_{1}+\omega_{2}\right)}\right\} \cos \left(3 \psi_{1}+2 \psi_{2}\right) \\
& -\left\{\frac{\alpha_{2} A_{1}^{3}}{8 \omega_{2}\left(3 \omega_{1}-\omega_{2}\right)}\right\} \cos \left(3 \psi_{1}-2 \psi_{2}\right) \\
& -\left\{\frac{3 \alpha_{3} A_{1}^{2} A_{2}}{16 \omega_{2}\left(\omega_{1}-\omega_{2}\right)}\right\} \cos \left(2 \psi_{1}-3 \psi_{2}\right) \\
& -\left\{\frac{3 \alpha_{3} A_{1}^{2} A_{2}}{16 \omega_{2}\left(\omega_{1}-\omega_{2}\right)}\right\} \sin \left(\psi_{1}-2 \psi_{2}\right)
\end{aligned}
$$




$$
\begin{aligned}
& -\left\{\frac{3 \alpha_{4} A_{1} A_{2}^{2}}{16 \omega_{2}\left(\omega_{2}-\omega_{1}\right)}\right\} \cos \left(2 \psi_{2}-\psi_{1}\right) \\
& +\frac{1}{2 \omega_{2}}\left\{\frac{1}{\Omega_{s}-\omega_{1}-\omega_{2}}\right\} A_{1} g_{1 s} \cos \left(\psi_{4}-\psi_{1}\right) \\
& -\left\{\frac{1}{4 \omega_{2}\left(\Omega_{s}+2 \omega_{2}\right)}\right\} A_{2} g_{2 s} \cos \left(\psi_{4}+\psi_{2}\right) \\
& -\left\{\frac{1}{4 \omega_{2}\left(\Omega_{s}+2 \omega_{2}\right)}\right\} A_{2} g_{2 s} \cos \left(\psi_{4}+3 \psi_{2}\right) \\
& -\frac{1}{4 \omega_{2}}\left\{\frac{1}{\Omega_{s}+2 \omega_{2}}+\frac{1}{\Omega_{s}}\right\} A_{2} g_{2 s} \cos \left(\psi_{4}+\psi_{2}\right) \\
& -\frac{1}{2 \omega_{2} \Omega_{s}} A_{2} g_{2 s} \cos \left(\psi_{4}-\psi_{2}\right) \\
& \left.-\left\{\frac{1}{4 \omega_{2}\left(\Omega_{s}+2 \omega_{2}\right)}\right\} A_{2} g_{2 s} \cos \left(\psi_{4}+3 \psi_{2}\right)\right],
\end{aligned}
$$

which is in excellent agreement with the solution obtained using the method of multiple scales and defined by (2.14).

5. Numerical results and discussion. Frequency-response (2.17) are nonlinear algebraic equation in the amplitudes $a_{1}$ and $a_{2}$. These equations are solved numerically using bisection method [10]. The numerical results are ploted in a group of Figures 5.1a, 5.1b, 5.2a, 5.2b, 5.3a, 5.3b, 5.4a, 5.4b, 5.5a, 5.5b, 5.6a, and 5.6b, which represent the variation of the amplitudes $a_{1}$ and $a_{2}$ with the detuning parameter $\sigma_{2}$ for given values of the other parameters.

In Figure 5.1a, each mode has one continuous curve and multivalued solution. Observe that the first mode reaches the minimum value at $\sigma_{2} \cong 3$ and the second mode reaches the maximum value to the same value of $\sigma_{2}$, and also it intersects in four points and these modes have stable and unstable solutions. The nontrivial solution has stable and unstable solutions represented on the frequency response curves by solid and broken lines and also the trivial solution has stable and unstable solutions represented by solid and dashed lines on the $\sigma_{2}$-axis. When $\mu_{1}$ takes the value 0.001 , we get the same frequency response curves without change in the magnitudes in both modes. The nontrivial and trivial solutions are unstable, as shown in Figure 5.1b. For increasing $\mu_{2}$, the bending of both modes are decreasing, which leads to decrease in the region of multivalued solutions. All nontrivial solutions are unstable and the stability of the trivial solution is decreasing, as illustrated in Figure 5.2a. As $\mu_{2}$ is increasing further, $a_{1}$ and $a_{2}$ has increasing and decreasing minimum and maximum values, and the multivalued region disappears. All the nontrivial solutions are unstable, and the zone of stability of the trivial solution is decreased, as shown in Figure 5.2b. Noting that, when $\mu_{2}$ takes the values -1 and -2 , respectively, we get the same Figures as 


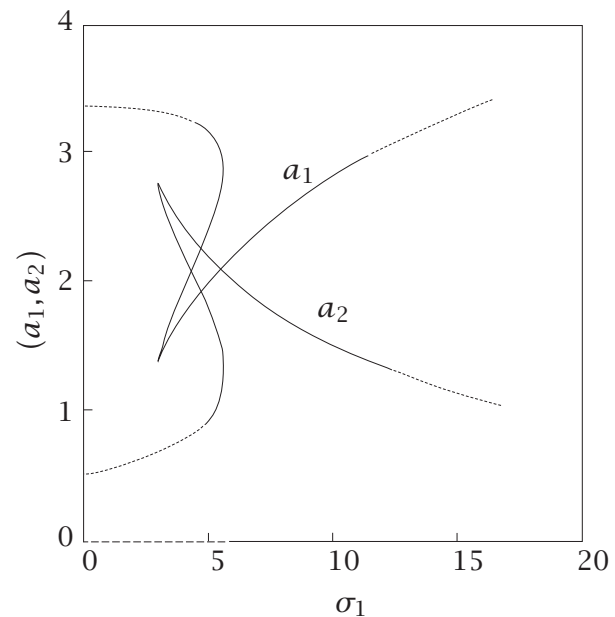

(a) Variation of the amplitudes of the response with the detuning parameter $\sigma_{2}$ for $\mu_{1}=0.1, \mu_{2}=$ $0.2, \alpha_{1}=\alpha_{2}=\alpha_{3}=1, \alpha_{5}=0$, $\sigma_{1}=2, \omega_{1}=0.2, \omega_{2}=0.6$, and $f_{1 r}=10$.

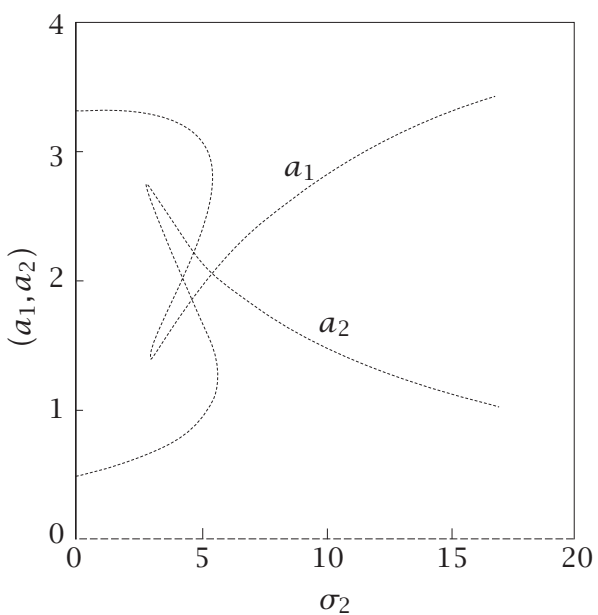

(b) Variation of the amplitudes of the response with the detuning parameter $\sigma_{2}$ for $\mu_{1}=0.001$.

FIGURE 5.1

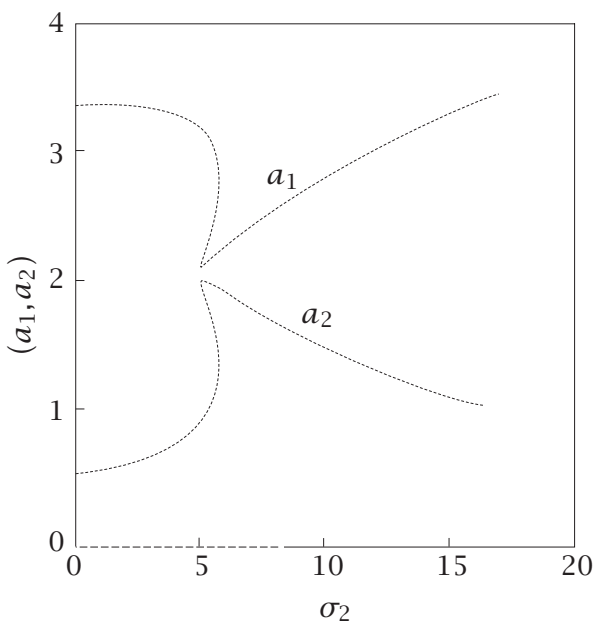

(a) Variation of the amplitudes of the response with the detuning parameter $\sigma_{2}$ for $\mu_{2}=1$.

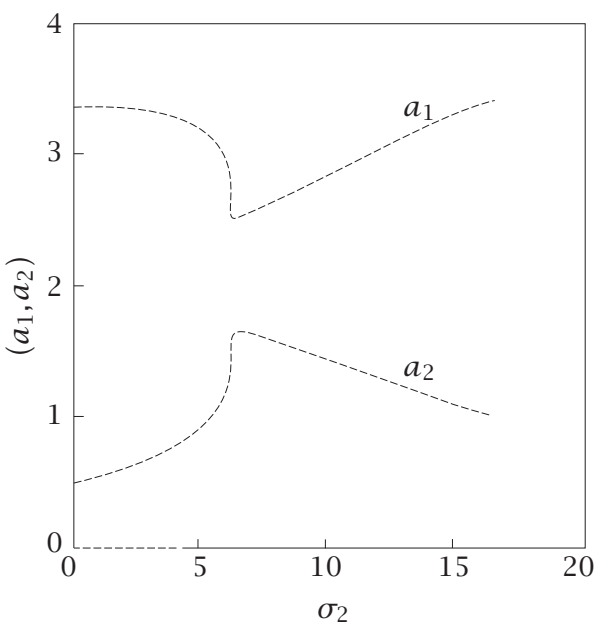

(b) Variation of the amplitudes of the response with the detuning parameter $\sigma_{2}$ for $\mu_{2}=2$. 


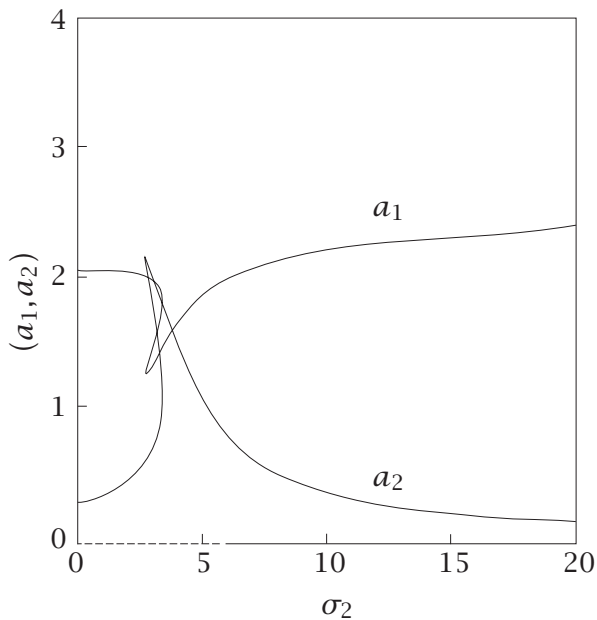

(a) Variation of the amplitudes of the response with the detuning parameter $\sigma_{2}$ for $\alpha_{1}=3$.

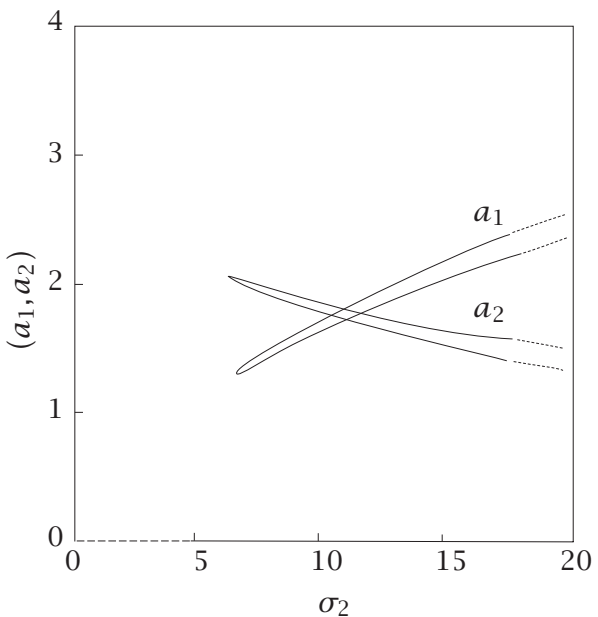

(b) Variation of the amplitudes of the response with the detuning parameter $\sigma_{2}$ for $\alpha_{3}=3.5$.

FIGURE 5.3

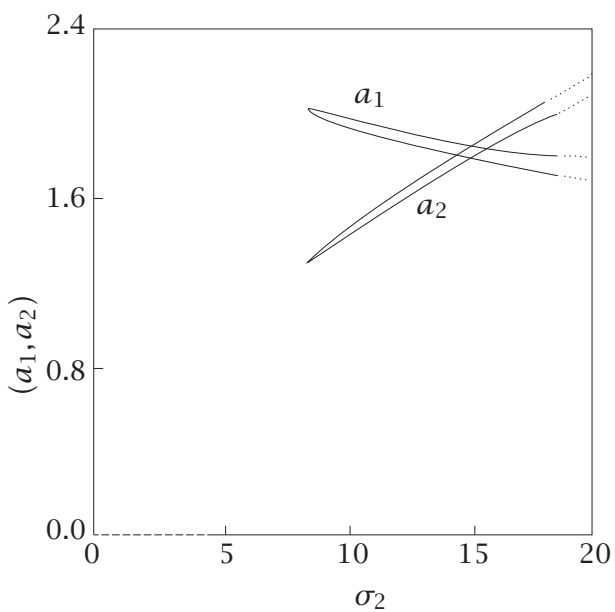

(a) Variation of the amplitudes of the response with the detuning parameter $\sigma_{2}$ for $\alpha_{3}=5$.

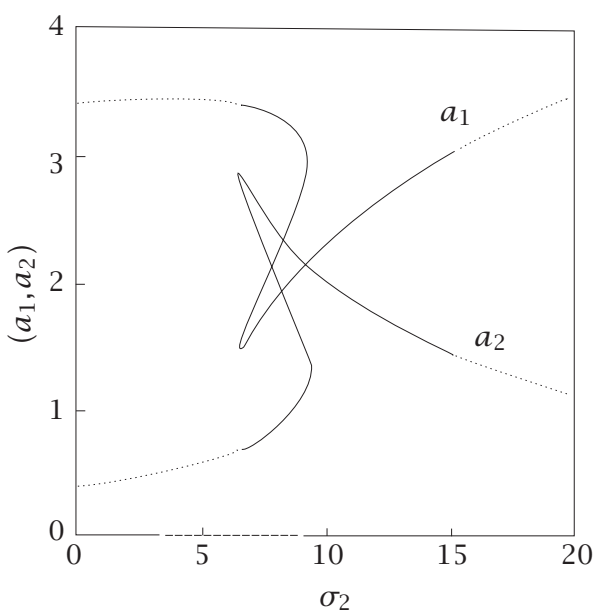

(b) Variation of the amplitudes of the response with the detuning parameter $\sigma_{2}$ for $\sigma_{1}=7$. 


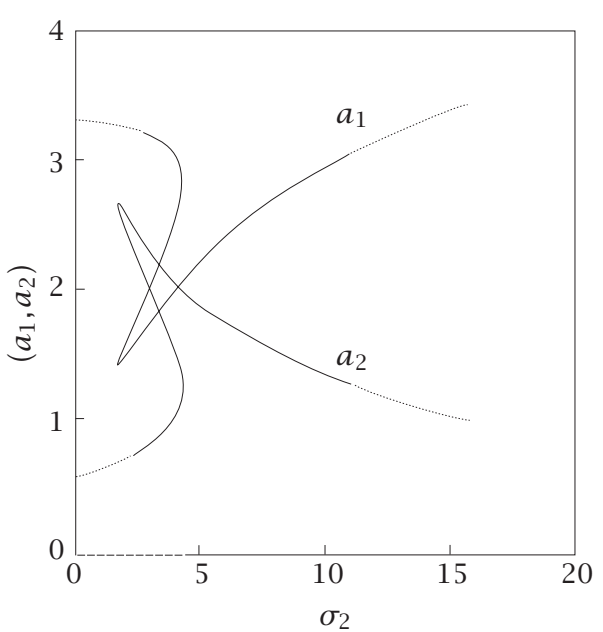

(a) Variation of the amplitudes of the response with the detuning parameter $\sigma_{2}$ for $\sigma_{1}=0.1$.

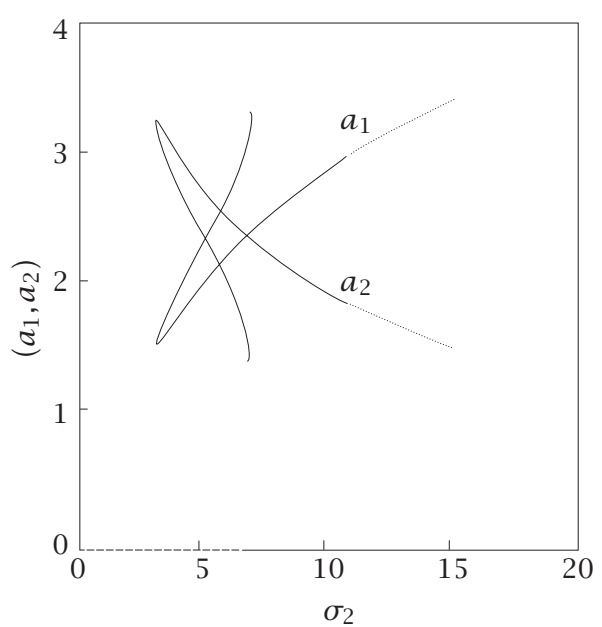

(b) Variation of the amplitudes of the response with the detuning parameter $\sigma_{2}$ for $f_{1 r}=13$.

FIGURE 5.5

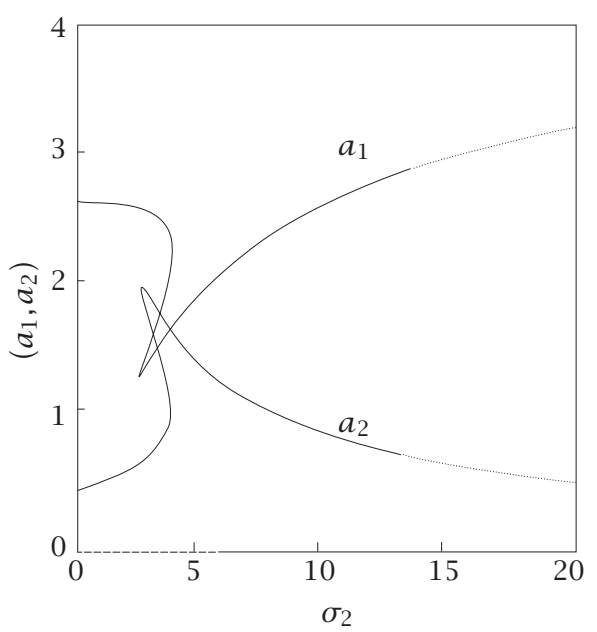

(a) Variation of the amplitudes of the response with the detuning parameter $\sigma_{2}$ for $f_{1 r}=6$.

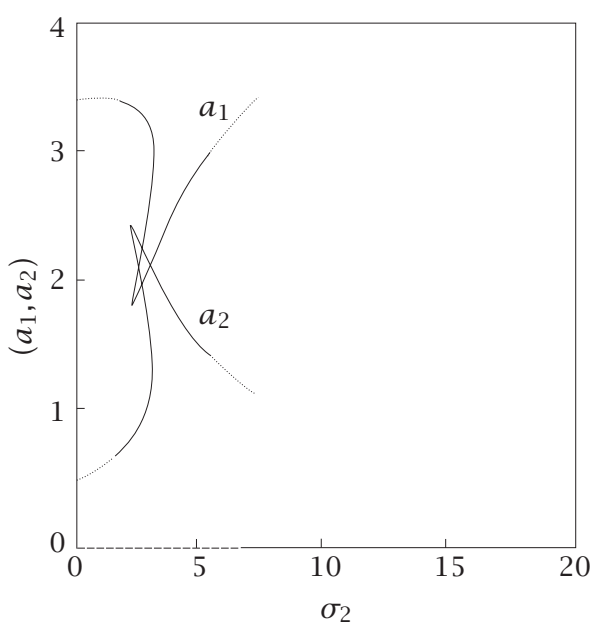

(b) Variation of the amplitudes of the response with the detuning parameter $\sigma_{2}$ for $\omega_{1}=0.5, \omega_{2}=$ 1.5 . 
Figures 5.2a and 5.2b without change in magnitudes for both modes and the trivial solutions are unstable. For increasing $\alpha_{1}$, both modes have decreasing magnitudes with the increase in the region of the stability and have decreasing minimum and maximum values and the region of definition is increased, as illustrated in Figure 5.3a. When $\alpha_{3}$ is increasing, both modes have stable magnitudes for large values of $\sigma_{2}$, and it has two semi-ovals which intersect in four points, and the zone of stability of the trivial solution is decreased, as shown in Figure 5.3b. For further increase of $\alpha_{3}$, the two semi-ovals have decreasing amplitudes which intersect in four points for large values of $\sigma_{2}$, as illustrated in Figure 5.4a. When the coefficient of detuning parameter $\sigma_{1}$ is increased, the region of the definition is increased and both modes have minimum and maximum values for large values of $\sigma_{2}$ and also have stable solutions for large values of $\sigma_{2}$, as shown in Figure 5.4b. Also as $\sigma_{1}$ decreasing further, both modes reach to decreasing minimum and maximum values for small value of $\sigma_{2}$, and it has stable solutions for small values of $\sigma_{2}$, as illustrated in Figure 5.5a. When the coefficient of the parametric excitation of the first mode $f_{1 r}$ is increased, each mode has one semi-oval, and the regions of definition and stability of trivial and nontrivial solutions are decreasing, as shown in Figure 5.5b. For further decrease of $f_{1 r}$, we observe that both modes have minimum and maximum values for small values of $\sigma_{2}$, and it has increased in the range of definition and stability, as illustrated in Figure 5.6a. For increasing the natural frequency of the first mode $\omega_{1}, a_{1}$, and $a_{2}$ have minimum and maximum values for small values of $\sigma_{2}$, and the regions of definition and stability are decreasing, as shown in Figure 5.6b.

6. Summary and conclusion. Two approximate methods are applied to construct a set of first order, nonlinear ordinary differential equations governing the modulation of the amplitudes and phases of oscillations. The applied method are the multiple scales and the generalized synchronization. Steady state solutions and their stability are studied for selected values of the different parameters. Numerical solutions are found by using bisection method, which are plotted in a group of Figures 5.1a, 5.1b, 5.2a, 5.2b, 5.3a, 5.3b, 5.4a, 5.4b, 5.5a, 5.5b, 5.6a, and 5.6b. The obtained results from both methods are in excellent agreement. The following conclusions can be deduced from analysis:

(1) the frequency response curves consists of one branch for each mode and the bending leads to multivalued solutions;

(2) all nontrivial solutions become unstable for increasing or decreasing the coefficients of damping terms of the first mode $\mu_{1}$ and the second mode $\mu_{2}$, respectively;

(3) when $\mu_{2}$ takes the values \pm 1 , we get the same frequency curves for these two values without change in amplitudes and also for the values \pm 2 , we get the same form without change in amplitudes;

(4) for increasing the coefficients $\alpha_{3}$ and $f_{1 r}$, we observe that each mode has one semi-oval and a given decrease in the region of definition;

(5) when the natural frequency of the first mode $\omega_{1}$ is increasing, the zones of definition and stability are decreasing. 


\section{REFERENCES}

[1] A. Abe, Y. Kobayashi, and G. Yamada, Two-mode response of simply supported rectangular laminated plateds, Internat. J. Non-Linear Mech. 33 (1998), no. 4, 675-690.

[2] A. K. Agrawal, J. N. Yang, and J. C. Wu, Non-linear control strategies for Duffing systems, Internat. J. Non-Linear Mech. 33 (1998), no. 5, 829-841.

[3] K. R. Asfar, A. H. Nayfeh, and D. T. Mook, Response of self-excite oscillation to multifrequency excitations, J. Sound Vibration 79 (1981), 589-604.

[4] __ Response of self-excited two-degree-of-freedom systems to multifrequency excitations, J. Sound Vibration 84 (1982), no. 2, 199-221.

[5] R. P. Ashworth and A. D. S. Barr, The resonances of structures with quadratic inertial nonlinearity under direct and parametric harmonic excitation, J. Sound Vibration 118 (1987), no. 1, 47-68.

[6] J. H. Balbi, Généralisation de la méthode de la moyenne, Internat. J. Non-Linear Mech. 8 (1973), 313-324 (French).

[7] A. M. Elnagar and A. F. El-Bassiouny, Response of self-excited three-degree-of-freedom systems to multifrequency excitations, Internat. J. Theoret. Phys. 31 (1992), no. 8, 1531-1548.

[8]___ Harmonic, subharmonic, superharmonic, simultaneous sub/super harmonic and combination resonances of self-excited two coupled second order systems to multifrequency excitations, Acta Mechanica Sinica 8 (1993), no. 1, 61-71.

[9] A. M. Elnaggar and G. M. Hamed-Allah, Determination of harmonic and subharmonic synchronization of a weakly non-linear conservative physical system, Bull. Fac. Sci. C Math., Assiut Univ. 10 (1982), no. 1, 115-129.

[10] C. F. Gerald, Applied numerical analysis, Wesly, 1980.

[11] N. N. Krylov and N. N. Bogliubov, Introduction to Non-Linear Mechanics, Princeton University, Princeton, 1947.

[12] A. Maccari, Dissipative bidimensional systems and resonant excitations, Internat. J. NonLinear Mech. 33 (1998), no. 4, 713-726.

[13] L. Meirovitch, Element of Vibration Analysis, McGraw-Hill Book Company, 1986.

[14] Z. Mojaddidy, D. T. Mook, and A. H. Nayfeh, Non-linear analysis of the periodic response of beams, Proceedings of the Sixth Canadian Congress of Applied Mechanic, 1977, pp. 387-388.

[15] A. H. Nayfeh, Introduction to Perturbation Techniques, Wiley-Interscience, New York, 1981.

[16] _ Combination resonances in the nonlinear response of bowed structures to a harmonic excitation, J. Sound Vibration 90 (1983), no. 4, 457-470.

[17] _ Combination tones in the response of single degree of freedom systems with quadratic and cubic nonlinearities, J. Sound Vibration 92 (1984), no. 3, 379-386.

[18] R. H. Plaut, N. HaQuang, and D. T. Mook, The influence of an internal resonance on nonlinear structural vibrations under two-frequency excitation, J. Sound Vibration 107 (1986), no. 2, 309-319.

[19] T. Yamamoto, K. Yasuda, and T. Nakamura, Combination oscillations in a nonlinear vibratory system with one-degree-of-freedom, J. Sound Vibration 92 (1974), 379-386, Bulletin of the Japanes Society of Mechanical Engineers.

A. F. El-Bassiouny: Mathematics Department, Faculty of Science, Benha University, BENHA 13518, EGYPT

Current address: Department of MATHEMATICS, Jubail College of EDUCATION FOR GiRLS, Jubail INDUSTRIAL CITY, 31961, P.O. BOX 12020, SAUdi ARABIA 


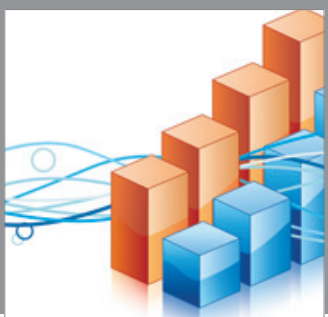

Advances in

Operations Research

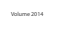

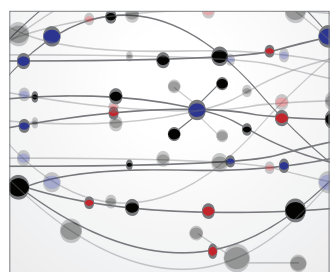

\section{The Scientific} World Journal
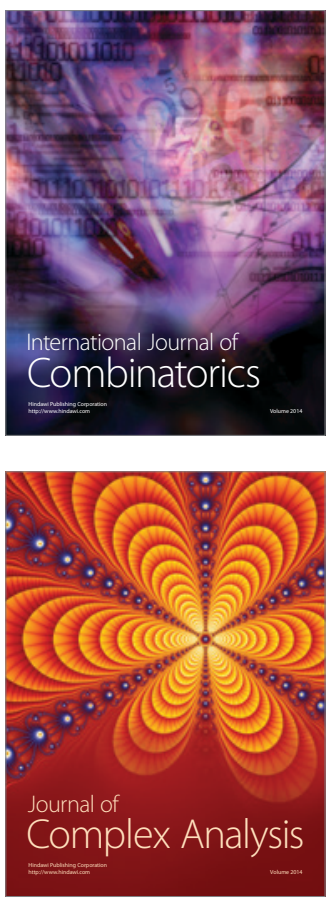

International Journal of

Mathematics and

Mathematical

Sciences
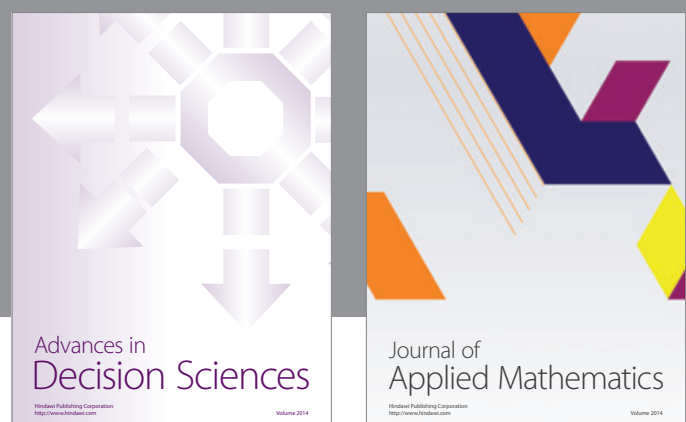

Journal of

Applied Mathematics
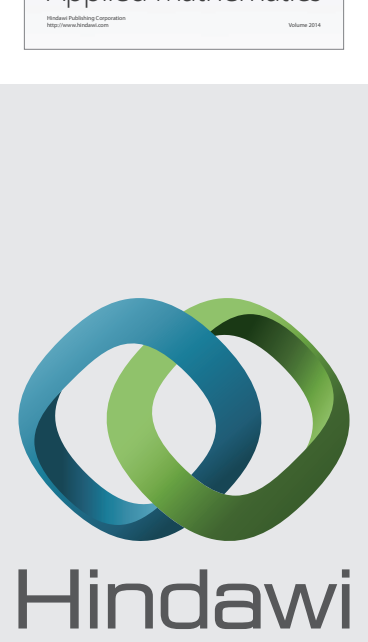

Submit your manuscripts at http://www.hindawi.com
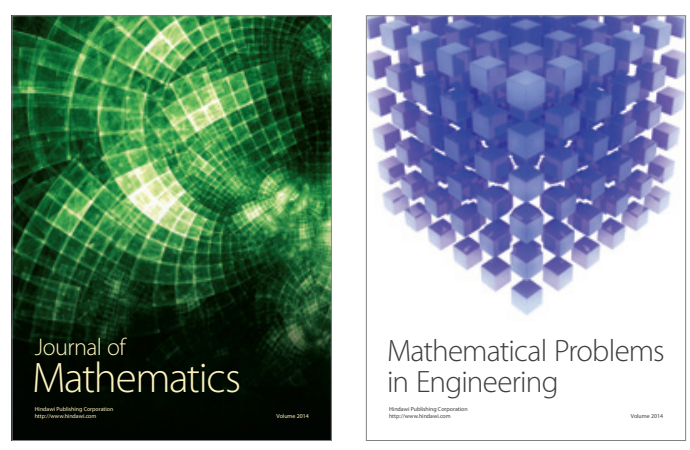

Mathematical Problems in Engineering
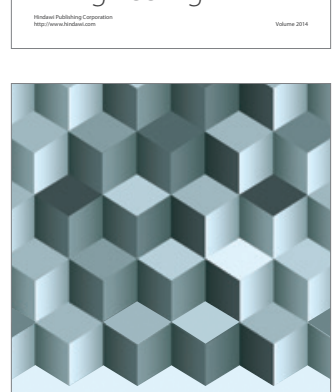

Journal of

Function Spaces
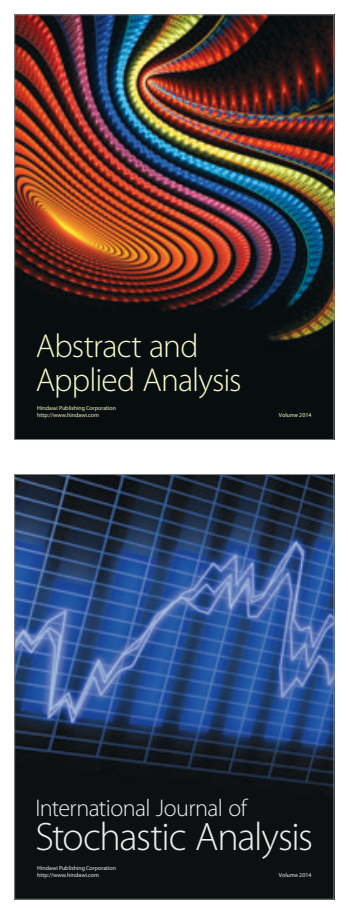

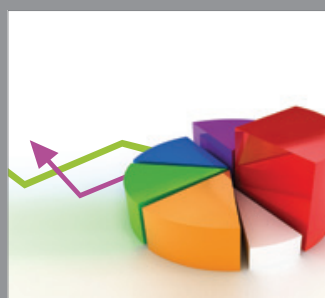

ournal of

Probability and Statistics

Promensencen
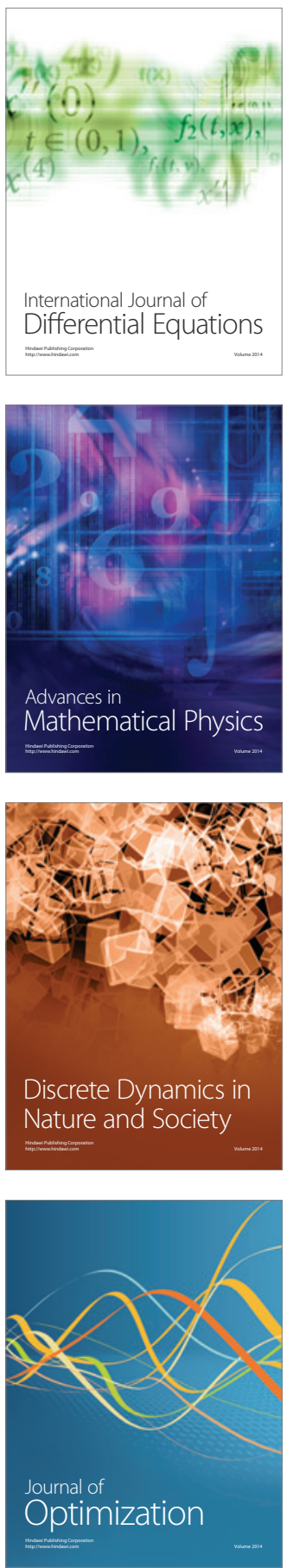